\title{
Perspective
}

\section{Computational Design of Thermally Activated Delayed Fluorescence Materials: The Challenges Ahead}

Yoann Olivier, Juan Carlos Sancho-García, Luca Muccioli, Gabriele D'Avino, and David Beljonne

J. Phys. Chem. Lett., Just Accepted Manuscript • DOI: 10.1021/acs.jpclett.8b02327 • Publication Date (Web): 28 Sep 2018

Downloaded from http://pubs.acs.org on September 29, 2018

\section{Just Accepted}

"Just Accepted" manuscripts have been peer-reviewed and accepted for publication. They are posted online prior to technical editing, formatting for publication and author proofing. The American Chemical Society provides "Just Accepted" as a service to the research community to expedite the dissemination of scientific material as soon as possible after acceptance. "Just Accepted" manuscripts appear in full in PDF format accompanied by an HTML abstract. "Just Accepted" manuscripts have been fully peer reviewed, but should not be considered the official version of record. They are citable by the Digital Object Identifier (DOI®). "Just Accepted" is an optional service offered to authors. Therefore, the "Just Accepted" Web site may not include all articles that will be published in the journal. After a manuscript is technically edited and formatted, it will be removed from the "Just Accepted" Web site and published as an ASAP article. Note that technical editing may introduce minor changes to the manuscript text and/or graphics which could affect content, and all legal disclaimers and ethical guidelines that apply to the journal pertain. ACS cannot be held responsible for errors or consequences arising from the use of information contained in these "Just Accepted" manuscripts. 


\title{
Computational Design of Thermally Activated Delayed Fluorescence Materials: The Challenges Ahead
}

Y. Olivier, ${ }^{1}$ J.-C. Sancho-Garcia, ${ }^{2}$ L. Muccioli, ${ }^{3}$ G. D'Avino, ${ }^{4}$ D. Beljonne ${ }^{1}$

${ }^{1}$ Laboratory for Chemistry of Novel Materials, University of Mons, Place du Parc 20, B-7000 Mons, Belgium.

${ }^{2}$ Departamento de Química Física, Universidad de Alicante, E-03080 Alicante, Spain.

${ }^{3}$ Dipartimento di Chimica Industriale "Toso Montanari", Università di Bologna, I-40136 Bologna, Italy, and Institut des Sciences Moléculaires, UMR 5255, University of Bordeaux, F- 33405 Talence, France.

${ }^{4}$ Institut Néel, CNRS and Grenoble Alpes University, F-38042 Grenoble, France.

Corresponding author: David.beljonne@umons.ac.be

\begin{abstract}
Thermally activated delayed fluorescence (TADF) offers the premise for all-organic light emitting diodes with quantum efficiencies competing those of transition metal-based phosphorescent devices. While computational efforts have so far largely focused on gas-phase calculations of singlet and triplet excitation energies, the design of TADF materials requires multiple methodological developments targeting among others a quantitative description of electronic excitation energetics, fully accounting for environmental electrostatics and molecular conformational effects, the accurate assessment of the quantum-mechanical interactions that trigger the elementary electronic processes involved in TADF, as well as a robust picture for the dynamics of these fundamental processes. In this perspective, we describe some recent progress along those lines and highlight the main challenges ahead for modeling, which we hope will be useful to the whole TADF community.
\end{abstract}

\section{TOC Figure:}

\section{Multiscale TADF molecular-based design}

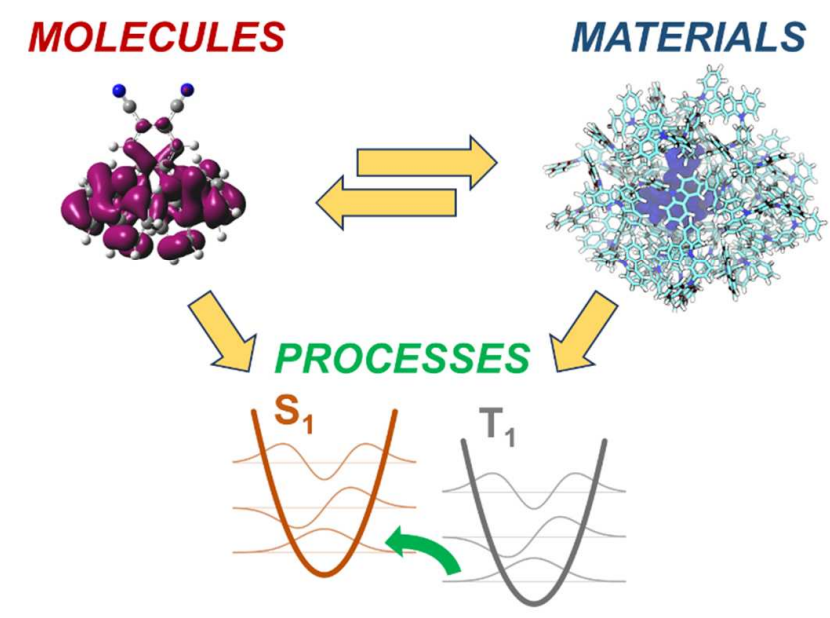


Organic light-emitting diodes (OLEDs) have emerged as a mature technology, reaching commercial applications in lighting and full-color displays. Delayed emission with characteristic spectra coinciding with prompt fluorescence but differing in the emission lifetimes has been observed in eosin solutions and has since been known as E-type fluorescence ${ }^{1}$. This is also referred to as Thermally Activated Delayed Fluorescence (TADF), a process that offers the premise to boost internal quantum efficiency (IQE) of electroluminescence beyond the $25 \%$ spin statistical limit. The demonstration of TADF in OLEDs has shifted the material design paradigm from phosphors containing rare and expensive transition metals towards all-organic compounds with reduced singlet-triplet exchange interactions.

(a)
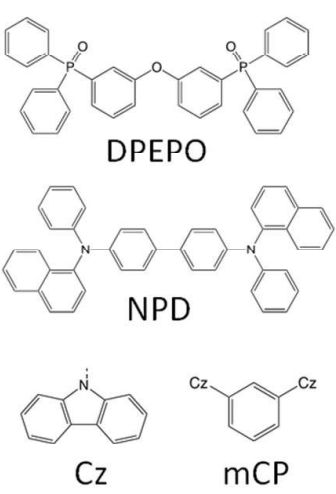

(b)

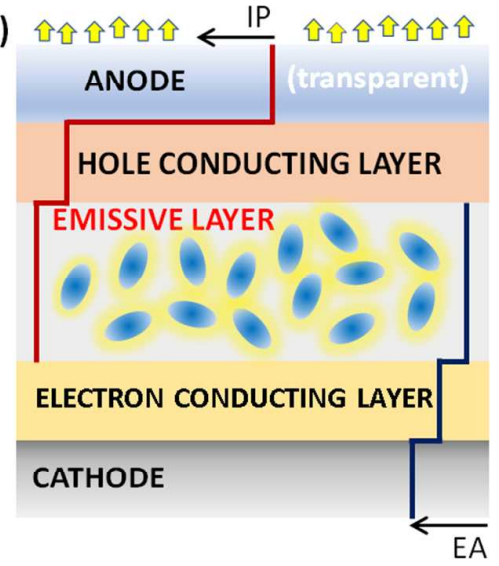

(c)
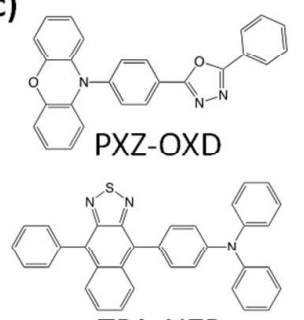

TPA-NZP

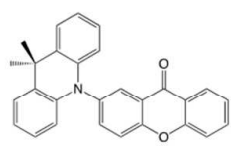

ACRXTN
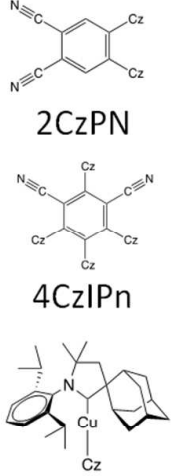

CMA2

Figure 1: (a) Chemical structures of some relevant host materials: Bis[2-(diphenylphosphino)phenyl] ether oxide (DPEPO), N,N'-Bis(naphthalen-1-yl)-N,N'-bis(phenyl)benzidine, (NPD) and 1,3-Di(9Hcarbazol-9-yl)benzene, 9,9'-(1,3-Phenylene)bis-9H-carbazole (mCP). Chemical structure of the carbazole (Cz) group. (b) Minimal sketch of a TADF-doped OLED, where electron and hole conducting layers serve also as hole and blocking layers, respectively, and as exciton blocking layer. Dark blue and red lines indicate typical levels for electron affinities and ionization potentials. This perspective focuses only on the emissive layer, where electron and holes recombine to form an exciton. The layer is constituted of a hole and electron conducting matrix (host), containing a few percent of TADF emitters (guest). The ideal host should possess EA (IP) values lower (higher) than the emitter. (c) Chemcial structures of emitters discussed in the text: phenoxazine-2,5-diphenyl-1,3,4-oxadiazole (PXZ-OXD), 4,5-di (9H-carbazol-9-yl) phthalonitrile (2CzPN), N,N-diphenyl-4-(9-phenylnaphtho-[2,3c][1,2,5]-thiadiazol-4-yl)aniline (TPA-NZP), 2,4,5,6-Tetra(9H-carbazol-9-yl)isophthalonitrile (4CzIPN), 3-(9,9-dimethylacridin-10(9H)-yl)-9H-xanthen-9-one (ACRXTN) and Cyclic (alkyl)(amino)carbene ligands copper-carbazole (CMA2). 
TADF emission is triggered by Reverse InterSystem Crossing (RISC) from the non-radiative triplet 'reservoir' states to radiative singlet states ${ }^{2}$, a process that is facilitated by a small energy splitting $\Delta \mathrm{E}_{\mathrm{ST}}$ between the lowest singlet $\left(\mathrm{S}_{1}\right)$ and triplet $\left(\mathrm{T}_{1}\right)$ excited states, and possibly assisted by the manifold of higher-lying triplet states $\left(T_{n} \text {, see Figure } 2\right)^{3-5}$. Hence, both prompt (from the lowest singlet) and delayed (from upconverted triplets) fluorescence decay channels add up in converting into light potentially all electrically generated excitons, irrespective of spin. The most common design strategy of TADF emitters consists in partitioning hole and electron densities over different spatial regions via electron donating (D) and accepting (A) units, often connected in a twisted conformation, hence reducing exchange interactions splitting singlets from triplets ${ }^{6-9}$. In actual cases, the excited states involved in TADF often turn out to be hybrid mixtures of charge transfer (CT) and local excitation (LE) diabatic states, with the amount of mixing prompted by vibronic coupling ${ }^{10,11}$. We refer the interested reader to a recently published review ${ }^{12}$ for a critical discussion of the chemical design rules that have emerged so far through the fruitful interplay between theoretical and experimental chemists. Despite some progress has been achieved, molecular design has largely relied on the calculation of excitation energies from the optimized ground-state geometry using timedependent density functional theory (TD-DFT), either for isolated molecules or assuming a continuum embedding (as for instance described using polarizable continuum models, PCM) ${ }^{13-15}$. The sole criterion for the selection of potential candidates for TADF is then the calculated exchange gap, $\Delta E_{S T}$, vertical or adiabatically, between the lowest singlet and triplet excitations. Besides raising a number of important technical questions regarding the accuracy of the predictions, this simplified view of the TADF mechanism might considerably bias the engineering rules, as it neglects potentially important effects such as the specifics of intramolecular conformation and intermolecular (hostguest) interactions on spin conversion dynamics in the solid state. This perspective aims at bringing a comprehensive view on the modeling of TADF emitters and at highlighting the challenges ahead. For being predictive, we argue that TADF-oriented computational models should include in an integrated framework $^{10}$ :

1) An accurate description of the singlet and triplet excited-state manifolds in terms of their relative energy and detailed nature, including coupling to relevant vibrational degrees of freedom;

2) A reliable evaluation of spin-orbit and hyperfine couplings mediating (reverse) singlet-triplet intersystem crossing;

3) A proper embedding scheme to account for both structural and electrostatic effects in the emitting layer; 
4) A solver for excited-state dynamics that includes electron-phonon and spin-mixing interactions on an equal footing.

In the following, the needed methodologies to reach these ambitious targets are critically reviewed and discussed, going all the way from a quantum-chemical description of the molecular TADF building blocks to the simulation of the corresponding solid-state materials using combined quantum-classical methods.

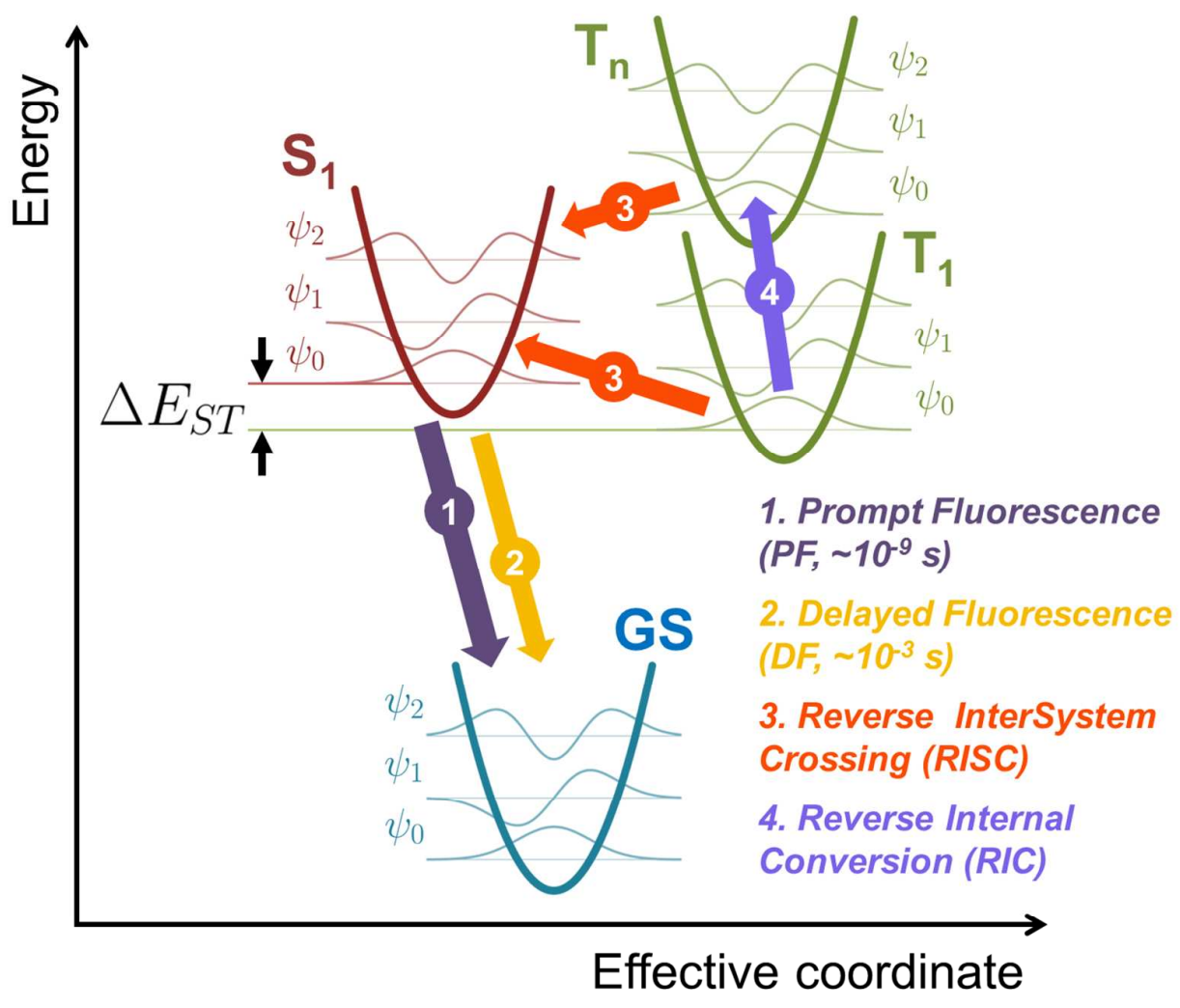

Figure 2: Illustration of the electronic states involved in TADF, i.e. ground (GS), singlet $\left(\mathrm{S}_{0}\right)$ and triplet $\left(T_{0}, T_{n}\right)$ excited states along an effective vibrational coordinate. The elementary steps leading to prompt (PF) and delayed (DF) fluorescence are illustrated as arrows. Low-quanta vibrational wavefunctions, which promote reverse intersystem crossing (RISC) and reverse internal conversion (RIC), are also shown. 
Generally speaking, the set of molecules acting as hosts or emitters share a common feature, that is, a relatively large size compared to those molecules constituting the datasets used for historically benchmarking $a b$ initio methods ${ }^{16-18}$. We focus here on modern variants of $a b$ initio theories for excited states, particularly for calculating vertical transition energies to the lowest excited-state of singlet and triplet spin symmetry, $\Omega\left(\mathrm{S}_{1}\right)$ and $\Omega\left(\mathrm{T}_{1}\right)$ respectively, also applicable to the whole manifold of $S_{n}$ an $T_{n}$ states. Although in principle superior to all other amenable approaches, due to the unfavorable scaling with system size $(N)$ of the family of methods based on iterative coupled-cluster (CC) equations (), their application to TADF compounds have been severely limited and only recently some examples applying the hierarchy of CC-based methods to small molecules have appeared ${ }^{19}$. Actually, for CC-based methods, the hierarchy is CC2 $\left(N^{5}\right)<\operatorname{CCSD}\left(N^{6}\right)<\operatorname{CC} 3\left(N^{7}\right)<\operatorname{CCSDT}\left(N^{8}\right)$, concerning both computational scaling with system size $(\mathrm{N})$ and expected accuracy. Note also that CC2 (CC3) is an approximation to CCSD (CCSDT) that has been developed with the prediction of excited-state energies as the main focus, and that all these variants are based on linear-response theory. On the other hand, EOM-CCSD $\left(N^{6}\right)$ is based on a slightly different theoretical frame, though it leads to similar excitation energies (yet different transition dipole moments).

The accuracy of the methods is very high, e.g. CCSDT excitation energies are within a few tens of meV w.r.t. $\mathrm{FCl}$, but they are only amenable to very small molecules, hence not applicable for most TADF chromophores. Yet, simplified versions of second-order wavefunction methods, and thus with a reduced formal scaling of $O\left(N^{5}\right)$ with respect to both canonical equations and higher-order methods such as CCSDT, are available. We mention, among them, linear-response CC2 and ADC(2) as emerging cost-effective and accurate methods ${ }^{20}$. Note that in the case of $\mathrm{CC} 2$, due to the fact that the full expression for double amplitudes is retained only at first-order, the method behaves better for single excitations and tends to overestimate excitations energies for doubly excited states. The $A D C(2)$ method suffers from the same inherent limitation for double excitations, and the corresponding $A D C(3)$ is too costly for routine calculations, although it leads to reliable benchmarks. However, the extended $A D C(2)-x$ version provides an overall improvement for the description of excited states with double-excitation character. An interesting compromise between accuracy and computational cost is provided by the spin-component-scaling (SCS-)CC2 method, through the introduction of scaling factors to the same-spin and opposite-spin contributions of the second-order correlation energy, leading thereby a better performance for excited states ${ }^{21}$. There are other methods that allow, by construction, $n$ - or multi-electron excitations, that is by promoting up to $n$ electrons into an active window of $m$ molecular orbitals, as in RAS- or CASSCF/CASPT2 methods. However, computational limitations preclude the use of large $(n, m)$ active spaces ideally including all relevant $\pi$ and $\pi^{*}$ orbitals. We finally mention many-body Green's function methods such as the GW plus Bethe- 
Salpeter equation (BSE) formalisms, also explored recently within this context with some success ${ }^{22,23}$. We shall, however, remark that full BSE suffers the issue of triplet instability, much as TD-DFT does ${ }^{24}$ (see below).

As other possible caveats for these calculations, we here mention: (i) the very costly calculation of excited-state geometries and adiabatic values for $\Omega\left(S_{1}\right)$ and $\Omega\left(T_{1}\right)$, which are important figures when dealing with light emission; and (ii) the marked dependence (i.e. slow convergence) with basis sets size expected for these methods and the possible need to include diffuse functions. This has historically prompted the use of more cost-effective methods, despite the fact that the computational efforts needed for $a b$ initio calculations can be reduced by using resolution-of-theidentity $^{25,26}$, or density-fitting techniques ${ }^{27}$. To conclude, so far, wavefunction-based methods are not routinely used for TADF applications and TD-DFT is usually preferred. However, they offer a robust framework to tackle challenging issues, such as assessing the contribution to RISC of higher-lying singlet and triplet excited states ${ }^{26}$ or modeling multiple resonance effects ${ }^{28,29}$.

Due to the need to handle large molecular sizes and to achieve a good trade-off between accuracy, scaling, and feasibility of the calculations for the fast screening of compounds, (Time-Dependent) Density Functional Theory, (TD-)DFT methods are by far the most employed computational tools in the TADF community. However, contrarily to what was initially thought, the reliability of the results depends not only on the functional choice for the time-dependent part, but also on the whole computational protocol employed including geometrical and basis sets issues, as well as on the chemical nature of the target molecule. We focus next on excited-state properties of emitters, for which a relatively wide body of information is available from the recent literature.

Earlier calculations combined the use of the B3LYP functional with a moderate basis set such as 6$31 G^{*}$ to obtain ground-state geometries and dissect the spatial shape and energy location of the frontier molecular orbitals ${ }^{30,31}$. When applied to the calculation of vertical transition energies to the lowest excited state of singlet and triplet spin symmetry and their difference dubbed as $\Delta E_{S T}^{V}$, the results were found to critically depend on the weight, or proportion, of the exact-like exchange introduced into the hybrid functional (typically ranging from 5 to $40 \%$, namely $20 \%$ for the widely used B3LYP model) ${ }^{32}$. Note that any general protocol should give accurate excitation energies for the lowest singlet and triplet excited states, without relying on any error cancellation and without limiting the focus to the magnitude of $\Delta E_{S T}^{V}$ as unique target. Namely, a system- and state-dependent procedure should be avoided, as extension to excited states beyond $S_{1}$ and $T_{1}$ might be problematic. Since organic molecules are known to benefit from higher-than-defaults (e.g. default values for 
B3LYP and PBEO are $20 \%$ and $25 \%$ respectively) weights of the exact-like exchange, other functionals like M06-2X have also been used with some success up to now ${ }^{33}$.

A major breakthrough was found after imposing the Tamm-Dancoff approximation (TDA) for the solution of the full TD-DFT equation ${ }^{34}$, which improves the accuracy of routine calculations. This is especially true for $T_{1}$ states where TDA cures for the triplet instability problem and, as a result, yields improved $\Delta E_{S T}^{V}$, values. Note that the Thomas-Reiche-Kuhn sum rule for oscillator strengths (i.e. the sum of all oscillator strengths from a particular state to all other equals the number of electrons in the system) is no longer fulfilled with TDA-DFT, as it happened with CIS too. That violation can lead to inaccurate oscillator strength distribution, and thus precludes a state-by-state quantitative comparison of oscillator strength values between theories fulfilling (i.e. TD-HF and TD-DFT) or violating the rule. The PBEO functional was also applied with some correction for dispersion, i.e. $D 3(B J)$, intended mostly to provide more accurate ground- and excited-state geometries after including non-covalent (intramolecular) effects, and used with large basis sets, i.e. def2-TZVP, to estimate $\Omega\left(\mathrm{S}_{1}\right)$ and $\Omega\left(\mathrm{T}_{1}\right)$ energies at the (nearly) complete basis set limit. However, while accuracy reaching 0.1-0.2 eV can be achieved for $\Delta E_{S T}^{V}$ values ${ }^{13}$, studies using this computational protocol have not yet been extended beyond $S_{1}$ and $T_{1}$ states, and thus further efforts are still needed in this direction.

Another strategy is provided by range-separated hybrid functionals, i.e. CAM-B3LYP or $\omega B$ 97X as paradigmatic examples, in which the range-separation parameter may be fine-tuned for each compound $^{14,35}$, as well as for isolated or host-embedded emitters. In these range-separation models, the electron-electron interaction is split into two contributions, short- and long-range, treating each one at a different theoretical level (short-range often with a semi-local GGA exchange functional and long-range with exact-like exchange to obtain the desired correct asymptotic behavior). The first mention in literature was due to A. Savin et al. in $1997,{ }^{36}$ but for the coupling of multiconfigurational ab initio with DFT methods, with probably the first application to GGA exchange functionals by $\mathrm{K}$. Hirao et al. in $2001,{ }^{37}$ and popularized later by N.C. Handy et al. with the CAM-B3LYP method. ${ }^{38}$ Since the tuning of the $\omega$ parameter aims at accurately reproducing one-electron attachment/detachment energies, it also naturally leads to accurate HOMO-LUMO gaps and corresponding excited-state energies (relying heavily on that energy difference, to first order). However, more work is still needed to confirm the accuracy of these methods for higher-lying singlets and triplets.

$S_{1}$ and $T_{1}$ optimized excited-state geometries are now routinely computed from linear-response TD(A)-DFT. However, different options such as the use of UKS (for $T_{1}$ ) or ROKS (for $S_{1}$ and $T_{1}$ states) exist $^{39}$. Still, we stress that mixing different levels of theory such as TD(A)-DFT (for $S_{1}$ ) and UKS (for $T_{1}$ ) 
can lead, in some instances, to spurious negative values of $\Delta \mathrm{E}_{\mathrm{ST}}{ }^{14,40}$. The fast screening of TADF molecules would also benefit of low-cost TD-DFT based methods, such as e.g. sTDA-DFT or sTDA$\mathrm{xTB}^{41}$. It would also be desirable to extend these methods beyond one-electron effects. The use of a (D)-like correction introduced by double-hybrid methods, namely a MP2-like term added non selfconsistently to the standard TD-DFT treatment, or the coupling of DFT with $\mathrm{MRCl}(\mathrm{DFT} / \mathrm{MRCl}), \mathrm{are}$ among the envisioned possibilities, although the price to pay is a higher formal scaling, $O\left(N^{5}\right)$ in the case of double-hybrid functionals. While double-hybrid models appear robust for $\Omega(\mathrm{S} 1)$ values, the lack of implementations for the calculation of the corresponding $\Omega(T 1)$ values has precluded so far the evaluation of $\Delta E_{S T}^{V}{ }^{42}$. DFT/MRCl has been recently extended to deal with higher-order excitations in multichromophoric systems ${ }^{43}$, opening new possibilities for exploring light-emission mechanisms for which both singlet and triplet states are of importance, as well as their possible modulation by environmental effects ${ }^{44}$.

The need for quantifying the nature of the excited states involved in the TADF process has led to the development of a set of metrics able namely to gauge the CT versus LE character of the excitations, which is critically entangled with the molecular geometry and its fluctuations around equilibrium $^{10,43,45}$. The existing metrics can be classified into two groups, those based on molecular orbitals, be them KS or NTO, and those based on density differences. Note that the metrics are intended to be: (i) generally applicable, in the sense that they can be applied to any of the desired singlet or triplet excited states; and (ii) easily transferable, with the results not expected to heavily depend on the functional and/or basis set choice. However, the use of relaxed and unrelaxed density might introduce some difference from a quantitative standpoint ${ }^{46}$ as it was recently disclosed.

Table 1 summarizes the observables developed so far and their typical values for the limiting cases of pure CT and LE. As regards the first category of tools, the metrics $\wedge$ roughly measures the overlap between a pair of occupied $(i)$ and virtual $(a)$ orbitals involved in the ground-to-excited state $\operatorname{transition}^{47,48}$; other approaches, such as $\Delta r^{49,50}$, aim at giving an effective hole-electron separation during the excitation. On the other hand, for density-based descriptors, the $D_{C T}$ index is based on the barycenters of densities associated with an electronic transition ${ }^{51}$, while $\Phi_{S}$ refers to the overlap between the attachment/detachment densities, that is, the electron density removed/rearranged during the excitation ${ }^{52}$. In some cases, due to a spurious behavior of density functionals for CT states, low-lying but unreal (intruder) CT states can appear, which can be also identified and discarded on the basis of the evaluation of intermolecular electrostatic interactions, e.g. by the $\mathrm{M}_{\mathrm{AC}}$ metrics ${ }^{53}$. Despite the appearance of a few topological metrics in the last years, there is also convincing evidence that these are highly correlated, as recently shown for a large sample of molecules 
displaying CT excitations, ${ }^{54}$ which should facilitate comparison between different existing or future studies.

Table 1. Summary of the metrics most used and their limiting values in the case of pure CT and LE excitations.

\begin{tabular}{|c|c|c|c|}
\hline \multirow[b]{2}{*}{ Metrics } & \multirow[b]{2}{*}{ Description } & \multicolumn{2}{|c|}{$\begin{array}{l}\text { Distinction between the } \\
\text { nature of excited-states }\end{array}$} \\
\hline & & CT & LE \\
\hline$\Lambda_{i a}$ & $\begin{array}{l}\text { Overlap between the ia pair of the norms of molecular } \\
\text { orbitals }\end{array}$ & $\wedge \sim 0$ & $\wedge \sim 1$ \\
\hline$\Delta \mathbf{r}$ & $\begin{array}{l}\text { Coefficient-weighted hole-electron distance } \\
\text { between a set of orbital centroids }\end{array}$ & $\Delta r>2 \AA$ & $\Delta r<2 \AA$ \\
\hline $\mathrm{D}_{\mathrm{CT}}$ & $\begin{array}{l}\text { Distance between barycenters of density variations } \\
\text { (average between the corresponding barycenters) }\end{array}$ & $t-D_{C T}>1.6 \AA$ & $\mathrm{t}-\mathrm{D}_{\mathrm{CT}}<1.6$ \\
\hline$\Phi_{\mathrm{s}}$ & $\begin{array}{l}\text { Normalized overlap between attachment/detachment } \\
\text { densities }\end{array}$ & $\Phi_{S} \sim 0$ & $\Phi_{S} \sim 1$ \\
\hline
\end{tabular}

Metrics based exclusively on orbitals raise some concern. For instance, many-electron excited states might involve multiple pairs of orbitals, thus casting doubt on the meaning of the $\wedge$ value. Another example is the case of a symmetric D-A-D triad, where the hole and electron centroids can spuriously occupy similar spatial positions, hence leading to a vanishing value of $\Delta r$, despite a strong CT excitedstate character. $\mathrm{D}_{\mathrm{CT}}$ and $\Phi_{\mathrm{S}}$ overcome these limitations and appear as more universal metrics ${ }^{55}$.

Interestingly, the use of these metrics has clearly evidenced that the excited states involved in the TADF process are neither full CT or LE, as abusively claimed when discussing the photophysics of TADF emitters ${ }^{3,5}$, but most often feature a rather mixed CT-LE character ${ }^{7,10,56}$ (see Figure 3a). The amount of CT-LE mixing is controlled by the magnitude of the D-A electronic coupling, which is in turn governed by structural parameters (such as the torsion angle between the two moieties) ${ }^{7,10}$. While the $S_{1}$ excited state often displays a large $C T$ character (small $\Phi_{S}{ }^{7}$ and large $\Delta r^{13}$, see Figure 3a), especially in conformations with near orthogonal $D$ and $A$ moieties ${ }^{6}$, this is less true for the $T_{1}$ excited state. This arises from exchange interactions that stabilize localized triplets more than their singlet counterparts, thereby prompting a more intimate LE-CT mixing in the triplet manifold ${ }^{7,57}$.

From a practical point of view, the metrics presented in table 1 have been applied as a tool to rationalize the $\Delta \mathrm{E}_{S T}$ and oscillator strength values in TADF compounds. Especially, it has been evidenced that the nature of $T_{1}$ is the limiting factor in order to minimize $\Delta E_{S T}$. The nature of $T_{1}$ can 
be directly probed experimentally by extracting the Zero-Field Splitting (ZFS) parameters from Electron Spin Resonance (ESR) spectroscopy studies. The dominant dipole-dipole component to the ZFS parameters is indeed inversely proportional to the third power of the interspin distance ${ }^{58}$. The larger the CT character of the triplet excited state, the larger the effective electron-hole radius and spin-spin distance and the lower the ZFS parameter value. From a computational point of view, the ZFS parameter can be evaluated through unrestricted DFT calculations using EPR-II and EP-III basis sets that are optimized for the computation of hyperfine coupling constants ${ }^{59}$. Interestingly, a recent study of carbazolyl-dicyanobenzene based TADF emitters (2CzPN and 4CZIPN) has demonstrated that ZFS (as calculated at the UKS level) and $\Phi_{S}$ (obtained from TDA-DFT) values go in par, offering the possibility to confront experimental and theoretical metrics of the triplet excitations ${ }^{60}$ (see Figure $3 \mathrm{~b}$ ). As for singlet excitations, calculated oscillator strengths have been shown to correlate very well to $\Delta \mathrm{r}^{11}$ and overlap metrics such as $\Lambda_{i a}{ }^{61}$ and $\Phi_{\mathrm{S}}{ }^{7}$.

Finally, we mention that only some of the metrics above can be calculated with common quantum chemistry codes, while others require post-processing after the TD(A)-DFT run. More automated procedures, and further benchmarking, are definitively needed, as well as their integration in largescale computational codes.

(a)

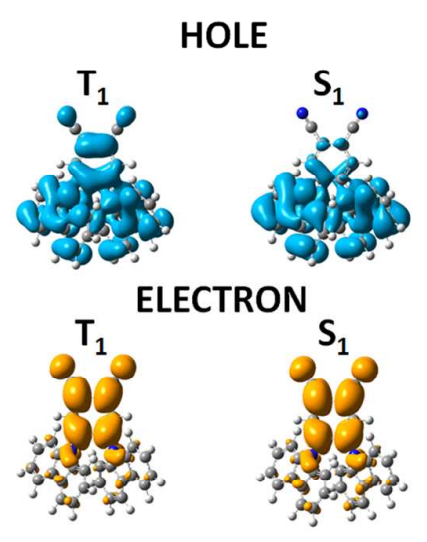

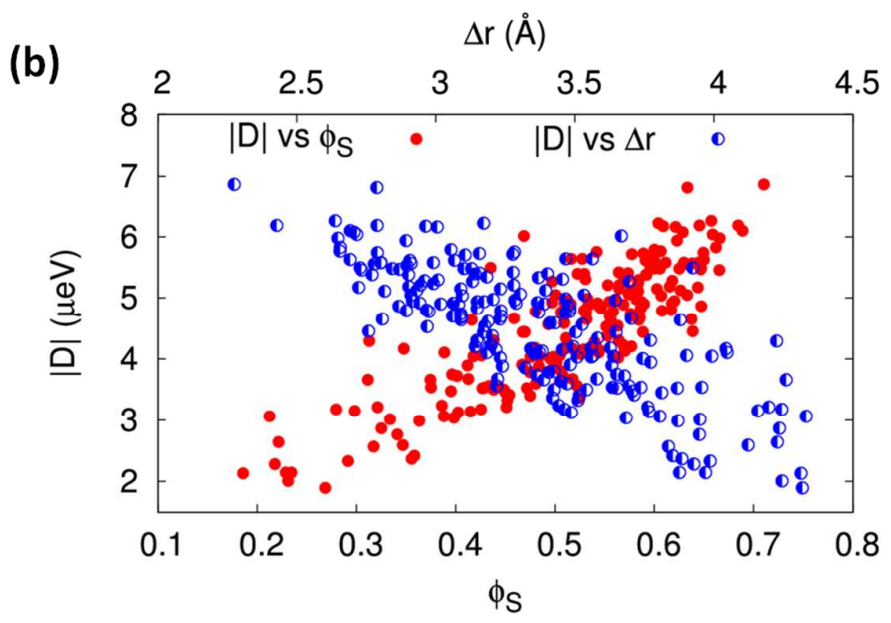

Figure 3: (a) Hole and electron densities calculated in the attachment/detachment formalism for $T_{1}$ and $S_{1}$ excited states for $2 \mathrm{CzPN}$. (b) Absolute value of the $T_{1}$ Zero-Field splitting parameter as a function of the overlap between the hole and electron densities $\phi_{\mathrm{S}}$ and the distance between the hole and electron densities centroids $\Delta r$, as calculated in the attachment/detachment formalism for $2 C_{z P N} T_{1}$ excited state. Reprinted and adapted with permission from reference ${ }^{10}$. Copyright 2017 by the American Physical Society

Non-radiative processes are playing a key role in determining the internal quantum efficiency of TADF-based devices. On the one hand, reverse intersystem crossing (RISC) and reverse internal 
conversion (RIC) are promoting the upconversion from the triplet to the singlet excited-state manifold. On the other hand, ISC favors the generation of triplets that could subsequently be recycled to $S_{1}$ or, because of their long lifetime, could decay through triplet-triplet or triplet-polaron annihilation, processes giving rise to device efficiency roll-off at high luminance. Of course, also nonradiative decays to the ground state from both the lowest singlet and triplet states are competing pathways reducing IQE. In the following, we distinguish between spin-conserving, namely (R)IC, triplet-triplet and triplet-polaron annihilation, and spin-non-conserving processes, namely ISC and RISC. Conformational effects on light emission and ISC and RISC are discussed thoroughly below.

Spin-orbit is a relativistic effect that is responsible for mixing orbital and spin degrees of freedom, thus allowing electronic states of different multiplicities to couple. Spin-orbit coupling naturally arises from the one-electron Dirac equation. Even though being of a fundamental interest, it cannot be solved exactly when considering large many-electron systems such as TADF molecules. Practical applications thus rely on perturbative approaches to non-relativistic electronic structure calculations. Among the most commonly reported approaches, we distinguish the zero-order relativistic approximation (ZORA) from the full Breit-Pauli Hamiltonian (including relativistic mass corrections and spin-orbit effects) and its mean-field approximation that are implemented in a number of softwares based on $\mathrm{CASSCF}^{62}$, TD-DFT and DFT-MRCI ${ }^{43}$ excited-state descriptions. The interest in spinorbit coupling calculations in the field of OLEDs dates back from the emergence of phosphorescent emitters containing heavy metal centers allowing for rapid ISC from $S_{1}$ to $T_{1}$ and radiative decay from $\mathrm{T}_{1}$ to the ground state (usually in the microsecond timescale regime). Because of the presence of these heavy atoms, spin-orbit coupling is usually large in phosphorescent emitters allowing for strong mixing between singlet and triplet excited states, while it is relatively weak (on the order of tenths or hundredths of meV) for TADF emitters made only of light elements. Still, the RISC mechanism is expected to be mainly driven by spin-orbit coupling with a small contribution of hyperfine coupling, as evidenced by electron paramagnetic resonance measurements ${ }^{9}$. This is further supported by transient electron spin resonance (TrESR) on 2CzPN and 4CzIPN carbazolyl-dicyanobenzene based TADF emitters, for which the observed absorption and emission patterns are characteristic of a spinorbit-driven ISC mechanism. Up to now, a systematic comparison between the different software and models has not been carried out yet, although it was shown that the full Breit-Pauli model and its mean field approximation lead to very similar spin-orbit coupling matrix elements for organic dyes $^{63}$. Already in the $60 \mathrm{~s}$, Mostafa El-Sayed ${ }^{64}$ highlighted the fact that spin-orbit coupling is vanishing when (R)ISC takes place between excited states with identical $\pi-\pi^{*}$ character. This is because the change in spin angular momentum must be compensated by a corresponding change in angular orbital momentum so that total angular momentum is conserved. As a result, only excited 
states with different spatial wavefunctions couple through spin-orbit coupling. A textbook example is benzophenone that sustains $n-\pi^{*}$ and $\pi-\pi^{*}$ electronic excitations coupled through large spin-orbit matrix elements ${ }^{65}$. Recently, this concept was put in practice in the context of TADF materials by associating the magnitude of the spin-orbit coupling to the difference in the nature of these excited states calculated based on the difference of normalized overlap between attachment/detachment densities, $\Delta \Phi S$, between $S_{1}$ and $T_{1} . . \Delta \Phi_{S}$ was calculated on molecular conformations taken from an amorphous morphology of pure films of 2CzPN and 4CzIPN. Spin-orbit matrix elements are found to correlate approximately linearly with $\Delta \Phi_{S}$, becoming vanishingly small for $\Delta \Phi_{S}=0$, namely in the case where $S_{1}$ and $T_{1}$ are nearly degenerate $\left(\Delta \mathrm{E}_{\mathrm{ST}} \sim 0\right)$ and exhibit both a strong and identical CT character, in line with the El-Sayed rules. Interestingly, because of its overall larger $\Delta \Phi_{S}$ offset, spin-orbit coupling is larger for $2 \mathrm{CzPN}$ than for $4 \mathrm{CzIPN}$ (see Figure 4b). Most importantly, we note that in the case of the carbazole derivatives studied in ${ }^{7}$ spin-orbit coupling and $\Delta \mathrm{E}_{\mathrm{ST}}$ have antagonistic evolution, such as a trade-off has to be found in order to maximize the rate of RISC (see Figure 4a).

Most importantly, we note that spin-orbit coupling and $\Delta \mathrm{E}_{\mathrm{ST}}$ have antagonistic evolution, such as a trade-off has to be found in order to maximize the rate of RISC (see Figure 3a).

(a)

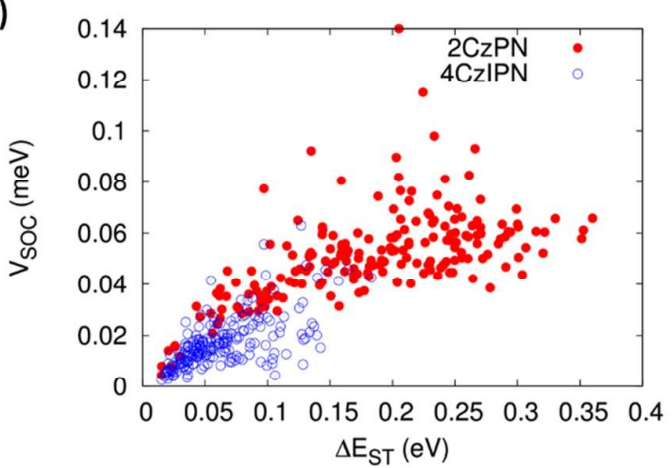

(b)

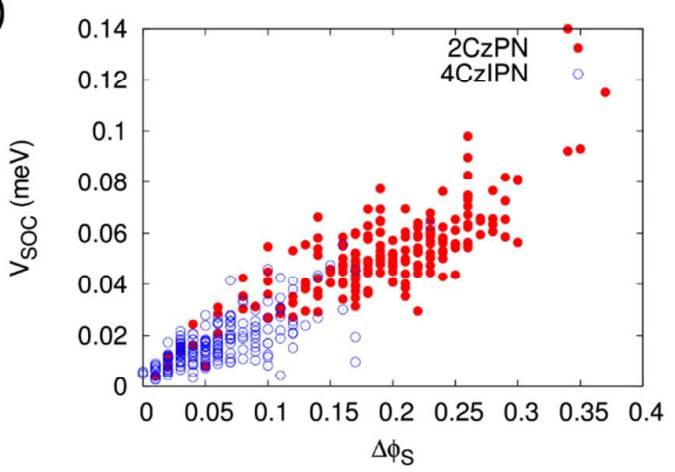

Figure 4: Spin-orbit coupling as a function of a) $\Delta \mathrm{E}_{S T}$ and b) $\Delta \Phi_{S}$ in $2 \mathrm{CZPN}$ (blue data) and 4CZIPN (red data), as sampled from a realistic amorphous morphology simulated with molecular dynamics. The approximately linear correlation between $V_{\text {SOc }}$ and $\Delta \Phi_{S}$ follows from the El Sayed rules, while the relationship between $\mathrm{V}_{S O C}$ and $\Delta \mathrm{E}_{S T}$ highlights the need for a trade-off between different parameters for the optimization of TADF performances. Reprinted with permission from reference ${ }^{10}$. Copyright 2017 by the American Physical Society.

Two different internal conversion mechanisms with contrasting effects are considered here. On the one hand, the reverse IC in the triplet manifold of excited states which is a thermally-activated process, is expected to assist RISC by promoting the formation of higher-lying triplet excited states, from which the conversion to the singlet manifold might occur more efficiently because it is 
associated with a larger exergonic character. This interconversion channel is expected to compete with the direct conversion from $T_{1}$ to $S_{1}$ and features smaller activation energies ${ }^{66}$. On the other hand, non-radiative recombination to the ground state should take place mainly from $S_{1}$ and contribute as the main monomolecular pathway to molecular excitation loss. Even though the two IC processes described above involve excited states of different spin multiplicities, the initial and final states are in both cases coupled through non-adiabatic couplings. The rate of internal conversion has been calculated in triphenylamine-thiadiazole molecule using a Fermi Golden rule formalism and considering non-adiabatic couplings for all relevant vibrational normal modes ${ }^{67}$. This approach, however, breaks down in the case internal conversion occurs at conical intersections, points of degeneracy between electronic states acting as dynamic funnels for radiationless transitions ${ }^{68,69}$.

In an OLED device, at high current, i.e. high hole and electron densities, triplets start accumulating in the device. Bimolecular processes such as triplet-triplet or triplet-polaron annihilation might occur, leading to an undesired roll-off behavior ${ }^{70}$. So far, no mechanistic picture has been proposed for these processes in the context of TADF materials. Triplet-triplet annihilation can proceed either via a virtual CT state or through a two-electron exchange mechanism, similarly to its reverse process, singlet fission ${ }^{71}$. The first attempts to include both processes in a device-like Kinetic Monte Carlo simulation have been based on phenomenological grounds considering that triplet-triplet (tripletpolaron) annihilation takes place when two triplet excitations (a triplet excitation and a charge) occupy neighboring sites ${ }^{72}$. Assessing annihilation rates from first principles remains very challenging since these processes involve the transient formation of high-lying electronic excitations with multiple-excitation character, the description of which demands highly correlated quantum-chemical methods often difficult to handle for large-size TADF emitters. A further challenge is that, since annihilation is a bimolecular process, its theoretical investigation requires the knowledge of the microscopic arrangement of emitters, so a further layer of calculations, as detailed in the next paragraphs.

With respect to the gas phase or implicit solvent calculations described so far, a step further towards the realistic modeling of TADF materials consists in taking into account explicitly the presence of surrounding host molecules. This is possible via a simple two-step multiscale scheme, by first employing classical (i.e. molecular mechanics) force fields and Molecular Dynamics (MD) or Monte Carlo $(\mathrm{MC})$ simulations to produce a realistic guess of the emitting layer morphology (see Figure 1 for a simplified scheme of a multilayer OLED architecture), ${ }^{73}$ and then performing electronic structure calculations on single molecules or clusters extracted from the simulated trajectories. This approach is considerably more expensive than a pure quantum mechanical-(QM-)based study, but its higher 
computational cost is compensated by the number of additional important effects occurring in real devices that can be accounted for, namely: i) sampling of many molecular geometries with a probability of occurrence depending on temperature (Boltzmann-weighted); ii) conformational changes and freezing induced by the solid matrix; and iii) inclusion of polarization and electrostatic effects. Besides, simulations predict the orientation distribution of the emitters and, more generally, provide the molecular positions of carriers and emitters, which are fundamental ingredients for device modeling, and in particular for kinetic simulations of electronic processes. ${ }^{74}$

Note that emitting layers are typically (meta)stable, disordered or partially ordered glasses, and host and emitter molecules themselves (Figure 1) have characteristic of organic glass-formers, being often constituted of a few, nonlinear rigid aromatic units interconnected by single rotatable bonds, allowing thus for a relatively large number of stable conformations. There are at least three routes to simulate amorphous morphologies of emissive layers, all of them essentially out of thermodynamic equilibrium. The simplest scheme starts with an NPT-ensemble MD simulation at temperatures and pressure high enough to obtain a fluid mixture of the desired composition, and subsequently cools the sample at room temperature and equilibrates it until average volume and total energy appear stable in time. ${ }^{10,75}$ A second, more costly approach imitates solvent annealing process, by starting from a concentrated solution of the target materials equilibrated at ambient conditions, from which molecules of solvent are progressively removed until a dry film is obtained. More recently, MD or MC simulations in which molecules are progressively inserted in the system and landed on a substrate, a scheme pioneered by some of us for the vapor deposition of organic crystalline semiconductors, ${ }^{76,77}$ have gained increasing popularity in the OLED research field. ${ }^{78-81}$ The origin of this trend is two-fold: on the one hand, real TADF-based active layers are in fact prepared most often by co-deposition of (at least) one host semiconductor and a guest TADF emitter, ${ }^{82}$ and on the other hand, since these films are amorphous, it is difficult to validate the simulation results versus experimental structural data, and it is tempting to believe that mimicking the experimental process could improve the quality of the predictions. Indeed, one of the open questions in the field is how important is the simulation procedure in determining the final morphology, and in turn how does it influence the calculated electronic properties. Experimentally, there is a large body of evidence suggesting that vapordeposited systems are in general more stable and dense than spin-coated ones prepared by solvent evaporation $^{82}$ or freezing from the liquid phase; ${ }^{83}$ however the exact shape of the potential energy landscape (see scheme in Figure 5 a for a pure material) not only does depend on the chemical nature of the host-guest system but also on their relative concentration. 
A further important result has recently emerged both from experiments ${ }^{78,84,85}$ and simulations: ${ }^{78-81}$ vapor deposited glasses can be to some extent orientationally anisotropic or, in other words, the orientation of the emitters in the active layer is not completely random. Since the specific orientation of emitter transition dipoles can strongly impact the outcoupling efficiency, ${ }^{86,87}$ it would be desirable to employ computer simulations in designing materials and setups apt at precisely controlling the orientation of the dyes, and then the direction of emitted light, in order to maximize the light output. The insurgence of anisotropy has been attributed to multiple factors, ranging from van der Waals interactions to dipole-dipole interactions and kinetic effects. These may be again system-dependent, but regardless of specific effects, it appears clear that a critical role is played by the orientation of the molecules at the growing interface with vacuum, as depicted in Figure 5b. Unfortunately, the current lack of knowledge about the relationship between all the simulation parameters (e.g. models, rates, temperatures, times, etc.) and the morphology obtained, so far does not allow to establish how general are the above-mentioned effects and to which extent they could be applicable to experimental results. Therefore, more systematic studies in this direction are urgent.

(a)

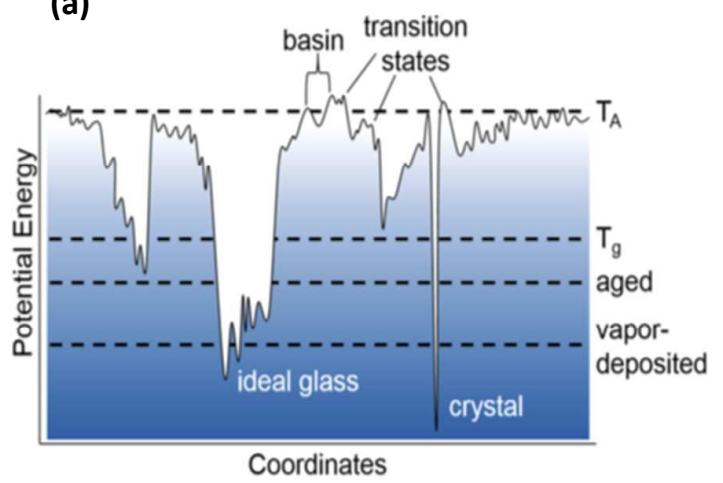

(b)

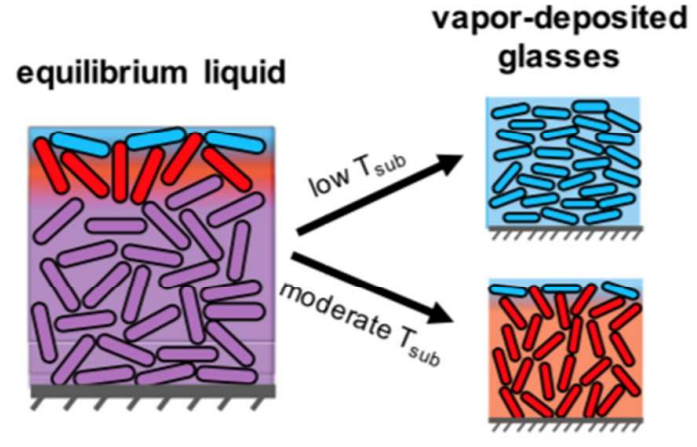

Figure 5: a) Schematic representation of the potential energy landscape of a glass forming system. TA is the temperature where non-Arrhenius dynamics are first observed in the liquid. Upon cooling at a constant rate, a temperature is reached at which molecular motions freeze and a glass is formed (glass transition temperature $\mathrm{T}_{\mathrm{g}}$ ). Aging slightly below $\mathrm{T}_{\mathrm{g}}$ allows some equilibration and lowers the potential energy. For some systems, much lower energies can be reached by physical vapor deposition with respect to aging or slow cooling. b) Possible origin of anisotropic molecular packing in vapor-deposited glasses of a rod-like molecule for which the free surface of the equilibrium liquid is anisotropic. ${ }^{78,88}$ The substrate temperature $T_{\text {sub }}$ determines the depth to which structure at the surface can equilibrate during deposition. The lowest portion of the growing film becomes trapped by further deposition. Also the interface with the solid substrate can be in principle anisotropic. Reprinted from reference ${ }^{83}$, with the permission of AIP Publishing. 
Another, maybe unavoidable, problem in comparing simulations and experiments is the enormous gap between the time scales accessible to simulations (microseconds) and the ones employed in the lab (minutes, hours). This difference might shift the balance between kinetic and thermodynamic effects, and may hamper the successful application of computer simulations to systems in which this balance is delicate. Concerning instead length scales, the mismatch between reality and atomistic models is much narrower, hundreds of nanometers versus tens or so. To reach the device scale, coarse-grained models can be employed, ${ }^{78,89}$ with the main disadvantage of some extra effort for the parameterization and complicated backmapping schemes to revert to the fully atomistic morphology, needed for electronic structure calculations. United-atom force fields can be an effective compromise, since the reduced number of centers allows a CPU time speed-up of about one order of magnitude ${ }^{90}$ and the backmapping to a full atom model is straightforward. Focusing on full-atom models, there is a raising awareness that the use of classical force field geometries for QM calculations may lead to uncontrolled approximations and systematic errors in the evaluation of electronic properties, e.g. $\mathrm{S}_{0}$ and $\mathrm{T}_{1}$ energies. ${ }^{10,75}$ In order to minimize this source of error, it would be beneficial to employ, in future studies, non-transferable force fields specifically tailored for reproducing not only the QM optimized geometry, but also the vibrational frequencies in the electronic state of interest. ${ }^{91}$

Radiative emission cross-sections, also quantified through the oscillator strength, are often very small in TADF molecules with close to orthogonal donor and acceptor moieties in their ground- or excited-state optimized geometries (see Figure 6b). Unless counterbalanced by specific intramolecular interactions such as hydrogen-bonds, ${ }^{61}$ most TADF molecules usually feature a twisted equilibrium conformation, which inevitably leads to a small overlap between the frontier $\pi$ conjugated orbitals of the donor and acceptor moieties. Fortunately, in TADF emitters based on D and $\mathrm{A}$ moieties connected through single bond, soft torsional modes are easily activated, generating a large spectrum of conformations at room temperature ${ }^{4,7}$ (see Figure 6a). These display significantly different absorption/emission energies and associated $\Delta \mathrm{E}_{\mathrm{ST}}$ values as the nature of the lowest singlet and triplet excited states (as probed through $\Phi_{\mathrm{S}}$ ) varies in time and space (see Figures $6 \mathrm{c}$ and $6 \mathrm{~d}$ ). Thermal excitation of these soft vibrational modes also results in broad and unstructured absorption and emission spectra. Thermal motion around the twisted equilibrium structures has a positive impact on luminescence, as it allows to sample conformations with larger overlap between the electron and hole wavefunctions, i.e. also larger oscillator strengths and radiative decay rates. ${ }^{20}$ In this case, emission of the TADF materials appears as a vibrationally-assisted process strongly coupled to soft (low-frequency) torsional modes. ${ }^{7}$ 
Also the RISC process, as claimed by Monkman and coworkers, ${ }^{3}$ can be seen as a spin-vibronic mechanism where spin mixing is dynamically gated by conformational fluctuations triggered by lowenergy torsional modes. The influence of torsional modes on excited-state dynamics is two-fold: (i) it brings excited states of the same spin multiplicity closer to each other, enhancing non-adiabatic coupling between the lowest singlet (triplet) excited states; ${ }^{92}$ (ii) it affects dynamically the nature of the singlet and triplet excited states involved in the TADF process in a way that the thermallyaveraged spin-orbit coupling is enhanced compared to its value at the equilibrium geometry. ${ }^{67,92}$ The role of vibrations on TADF dynamics can be modeled using different formalisms, either based on nonadiabatic molecular dynamics, such as mixed quantum-classical surface hopping methods ${ }^{93}$ or full quantum wavepacket propagation simulations, ${ }^{94}$ or relying on rate expressions derived from timedependent second-order perturbation theory (Fermi Golden Rule). ${ }^{67,92}$ These approaches usually include a set of preselected intramolecular vibrational modes for which the frequencies and displacements (electron-phonon couplings) are computed from first-principles. Alternatively, one can resort to MD simulations that allow sampling all vibrational modes classically, being intra- or intermolecular in origin, at once. In the case of low-frequency vibrations, classical and quantum approximations yield similar results for thermally averaged spin-orbit coupling and $\Delta \mathrm{E}_{S \mathrm{~T}}$ values. ${ }^{95} \mathrm{The}$ reorganization energy associated with high-frequency vibrations, i.e. mostly bond stretching, can be obtained from ground- and excited-state geometry optimization of the isolated molecules. These modes steer temperature-independent quantum tunneling effects and can be easily incorporated into rate expressions for ISC and RISC. For instance, by a careful analysis of the time evolution of the dihedral angles in 2CZPN and 4CZIPN, a characteristic time scale of about 1 ps has been inferred. This is fast compared to RISC, hence a large portion of the dihedral angles distribution is explored by the molecule before upconversion takes place, which confirms the truly dynamic nature of the RISC process. Considering thermally-averaged spin-orbit coupling and $\Delta \mathrm{E}_{S \mathrm{ST}}$ for the specific case of $4 \mathrm{CZIPN}$, (R)ISC rates calculated within the semi-classical Marcus theory were found to be in excellent agreement with experimental data. ${ }^{10}$

To close this discussion, we would like to briefly refer to studies pointing to the role of 'hard' modes. For instance, it has been shown that the displacement along a $\mathrm{C}=0$ stretching mode on the donor moiety of a xanthone-acridine D-A complex is able to bring in near resonance triplet ${ }^{3} \mathrm{CT}$ and ${ }^{3} \mathrm{LE}$ states, from which efficient RISC to ${ }^{1} \mathrm{CT}$ proceeds. ${ }^{96}$ Similarly, highly correlated wavefunction based calculations in carbene-metal-amide complexes suggest that the dynamic red shift observed experimentally is associated with changes in the carbon-nitrogen bond length and metal-carbonnitrogen bond angle within the carbene-metal-amide three-center core. Very interestingly, these changes reduce $\Delta \mathrm{E}_{\mathrm{ST}}$, while keeping unaffected the spin-orbit coupling and emission transition dipole 
moment from the singlet excited state, at odds with the initially proposed rotationally-assisted upconversion mechanism. ${ }^{45}$

(a)

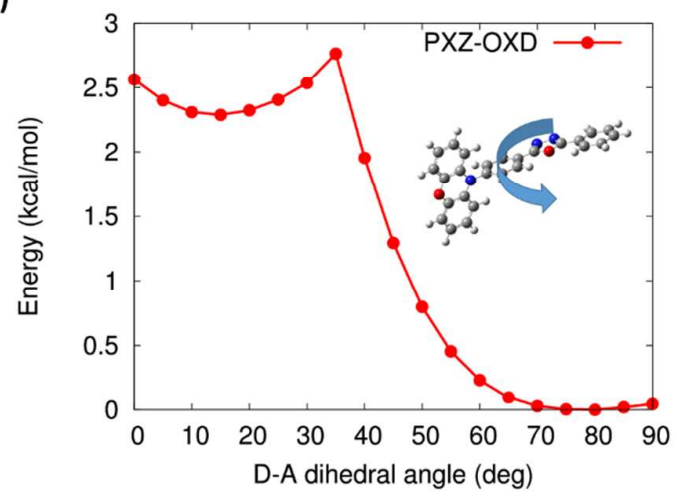

(b)

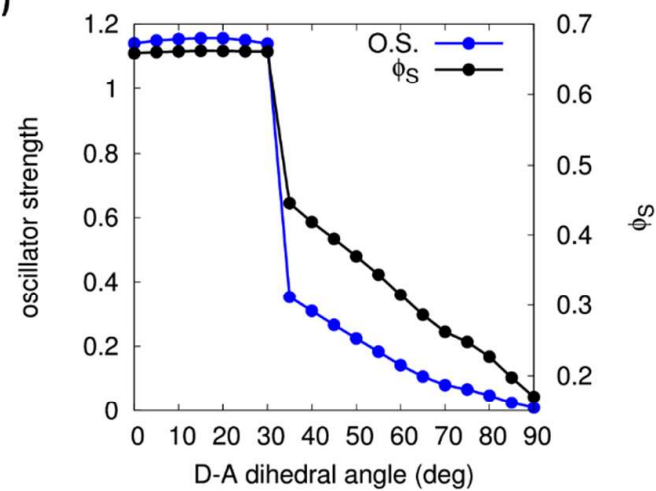

(c)
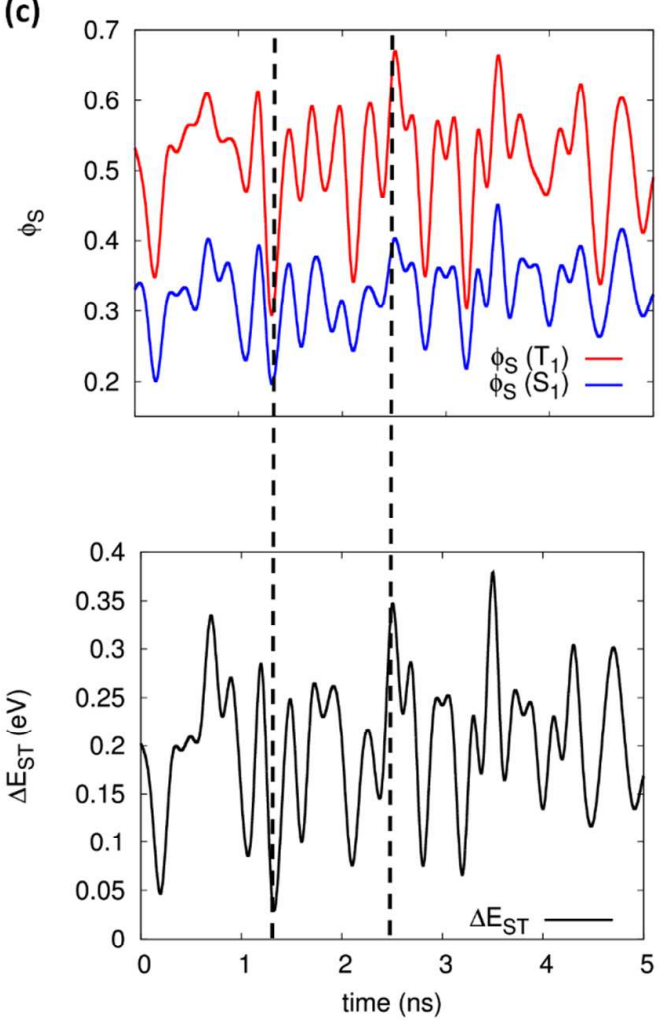

Figure 6: (a) Torsional energy profiles calculated at the PBE0-D3(BJ)/6-31G(d,p) level of theory with the PCM module for solvent (toluene) for PXZ-OXD. (b) Variation of $\triangle \mathrm{E}_{\mathrm{ST}}$ and and oscillator strength (O.S) as a function of the D-A torsion angle. (c) Time evolution of the $\Phi_{S}\left(S_{1}\right)$ and $\Phi_{S}\left(T_{1}\right)$ from electronic structure calculations performed along a molecular dynamics trajectory for 2CzPN. (d) Time evolution of the $\Delta \mathrm{E}_{S T}$ for $2 \mathrm{CZ}_{\mathrm{P} N}$. Vertical dashed lines highlight that $\Delta \mathrm{E}_{S \mathrm{~T}}$ is the largest (smallest) when the difference in $\Phi_{S}\left(S_{1}\right)$ and $\Phi_{S}\left(T_{1}\right)$ is the largest (the smallest). Reproduced from Ref. ${ }^{7}$ with permission from The Royal Society of Chemistry. Reprinted and adapted with permission from reference ${ }^{10}$. Copyrights 2017 by the American Physical Society.

Environmental effects are known to have a major impact on molecular excitations of charge transfer character, yet the implications on $\Delta \mathrm{E}_{S T}$ in TADF emitters has started to be appreciated only recently. The role of the environment is particularly subtle and important in systems where singlet and triplet excitations of CT and/or LE character are all close in energy and compete, while the energy window interesting for applications is of few tens of meV. Viable computational strategies consist in $\mathrm{QM} / \mathrm{MM}$ approaches, where the TADF emitter (QM system) is embedded in a classical medium that can be 
described either with PCM or with discrete schemes of atomistic resolutions. Both approaches have advantages and limitations that we briefly discuss in the following.

In this context, Brédas and coworkers systematically employed the PCM in their TD-DFT investigations of singlet-triplet splitting and spin-orbit matrix elements for different TADF molecules. ${ }^{14,56}$ Sun et al. specifically addressed the effect of the dielectric constant $(\varepsilon)$ on the nature of the excited states and on the single-triplet splitting by using optimally tuned range-separated hybrid functionals, whose range separation parameter $\omega$ was optimized for each value of $\varepsilon .{ }^{97}$ These calculations highlighted the role of the medium polarizability in stabilizing electronic configurations with large CT character, in turn affecting the CT-LE hybridization in singlet and triplet excitations. For instance, dipolar D-A molecules such as TXO-TPA and TXO-PhCz, which in the gas phase are prescribed to feature large $\Delta \mathrm{E}_{\mathrm{ST}}$ (ca. $0.5 \mathrm{eV}$ ) as a result of the large LE character of $\mathrm{T}_{1}$, become interesting for TADF applications in a typical organic matrix with $\varepsilon^{\sim} 3$. Indeed the medium polarization leads to excitations with large CT character and nearly non-overlapping hole and electron clouds and $\Delta \mathrm{E}_{\mathrm{ST}}$ below $0.1 \mathrm{eV} .{ }^{97}$ Marian et al. ${ }^{44}$ proposed a reinterpretation of the emission observed for carbene-metal-amide in both solution and film based on a combination of DFT/MRCI and PCM. Especially, they showed that solvent reorganization has to be taken into account when computing the delayed fluorescence spectrum, while it is not required when discussing prompt fluorescence occurring at early-timescale. Practically speaking, one needs to consider the relaxed density matrix of the excited state to calculate the solvent reaction field. Delayed fluorescence appears to be redshifted in comparison the prompt one in agreement with experiment. However, in glassy films, such a treatment is not needed because the medium reorientation is sterically hindered so that delayed fluorescence appears to be blue-shifted compared to solution. Along the same line, Penfold et al. ${ }^{75}$ have highlighted through a combination of MD and TD-DFT calculations that a blue shift in the delayed emission of D-A TADF emitters in films at the longest timescale does not result from host reorganization, and thus on specific host-guest interaction, but rather from a distribution of CT states with different emission energies. The prompt fluorescence is essentially governed by the higherenergy CT states that exhibit the largest hole-electron wavefunction overlap and therefore also oscillator strength. As for the delayed fluorescence, the early part of the signal appears to be redshifted in comparison to the prompt fluorescence since RISC occurs first through the lower-energy CT states that minimize $\Delta \mathrm{E}_{\mathrm{ST}}$. The late delayed fluorescence component then occurs through higher-lying but more emissive CT states, thereby rationalizing the blue-shift observed.

Beyond PCM, atomistic polarizable models aim at describing excitations of molecules in their specific environment, usually an amorphous matrix blending the charge transporting material and other emitters. Atomistic simulations of such blends are a prerequisite for these approaches, which also 
allow for the sampling over a statistical collection of molecule-environment configurations. Our group adopted this route to study environmental effects in carbazolyl-dicyanobenzene based TADF emitters, 2CzPN and 4CzIPN, employing atomistic microelectrostatic models parameterized from first principles. ${ }^{10}$ These calculations showed that the medium polarization, modeled with mutually interacting anisotropic polarizabilities, reacts differently to states of different CT character (see Figure 7a). For instance, $2 \mathrm{CzPN}$ shows a singlet excitation $\mathrm{S}_{1}$ that has larger $\mathrm{CT}$ character than the triplet $T_{1}$, and thus a larger electrical dipole, resulting in a stronger stabilization of the former by the environment polarization, finally leading to a reduction in $\Delta \mathrm{E}_{\mathrm{ST}}$ (see Figure $7 \mathrm{~b}$ ). This provides a general mechanism through which the embedding medium compensates for large $\Delta \mathrm{E}_{\mathrm{ST}}$ established at the molecular level, leading in some cases to negative $\Delta \mathrm{E}_{\mathrm{ST}}$ values. In addition, atomistic models do also account for the fact that molecules are distorted in real morphologies and experience the inhomogeneous electrostatic potential of the neighborhood. These phenomena affect the energies of the excited states within both the singlet and the triplet manifold of states, leading to inhomogeneous broadening in the solid matrix and to broad distributions of $\Delta \mathrm{E}_{\mathrm{ST}}$ values. Such a disorder can also break the symmetry of the molecule (e.g. localizing the hole of a CT excitation on a given $\mathrm{D}$ carbazole unit) and, most interestingly, have a dynamic nature, i.e. lead to a modulation in time of the nature of the relevant states (CT-LE hybridization) and of the $\Delta \mathrm{E}_{\mathrm{ST}} \cdot{ }^{10}$

(a)

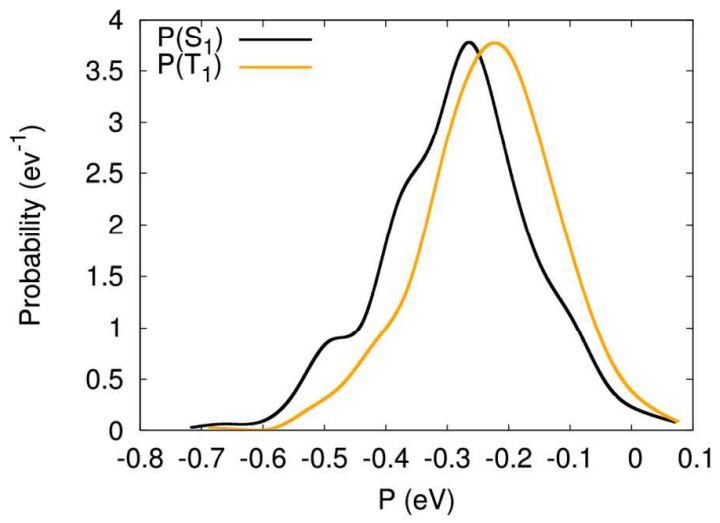

(b)

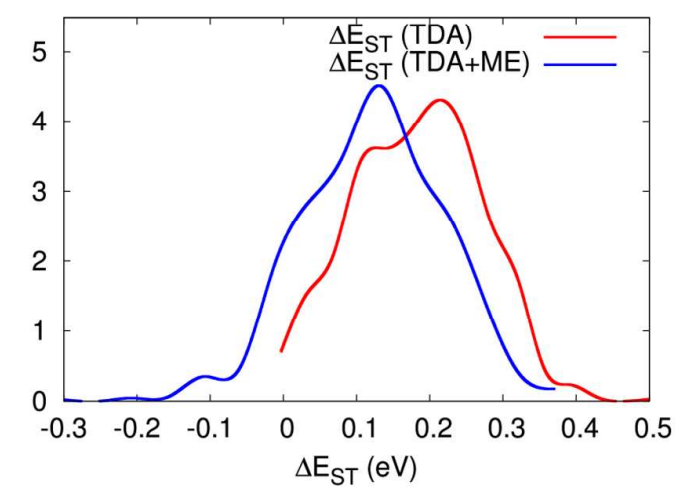

Figure 7: (a) Polarization energy distributions associated to $S_{1}$ and $T_{1}$ excited states. (b) Distributions of $\triangle \mathrm{E}_{\mathrm{ST}}$. Red and blue lines correspond to TDA-PBEO results in the vacuum and accounting for local dielectric effects, respectively. All data provided in this Figure refer to $2 \mathrm{CzPN}$. Reprinted and adapted with permission from reference ${ }^{10}$. Copyright 2017 by the American Physical Society. 
In summary, predictive modeling of TADF emitters requires an integrated multiscale approach able to capture the energetics and the dynamics of electronic excitations in realistic morphologies of OLEDs emitting layers. In combination with state-of-the-art experimental investigations (optical spectroscopy and device characterization), computational studies have already shed light on some key features of TADF, including:

- $\quad$ The excited states involved in TADF often feature mixed CT and LE character.

- The host influences $\Delta \mathrm{E}_{S \mathrm{~T}}$ through conformational and dielectric effects.

- $\quad$ RISC and light emission are dynamic processes assisted by intramolecular vibrations.

While some of the items above remain open to discussion, as they are likely material-specific, they also prompt new questions and challenges that need to be addressed when designing the next generation of TADF emitters. For instance, can one tune the nature of the excited states involved in the TADF process in order to speedup RISC? Can one take advantage of environmental effects to design molecules with negative $\Delta \mathrm{E}_{\mathrm{ST}}$ ? Can we expand the modeling approaches in order to account for all the mono- and bi-molecular radiative and non-radiative processes taking place in TADF-based OLEDs and identify host-guest combinations that would minimize annihilation and maximize pure and color-tunable light emission?

Clearly the answer to these questions can only be obtained in the scope of a multifaceted theoretical framework, where molecular and material properties are conjointly addressed and optimized. More specifically, we would like to end this perspective with modeling challenges inspired by a few selected opportunities from recent experimental investigations:

- Hyperfluorescent OLEDs. ${ }^{98}$ Here, TADF molecules act mostly as assistant dopants that drive the excitations towards a dye with narrow-line singlet emission. To further improve what is referred to as the $4^{\text {th }}$ generation OLEDs, a microscopic picture for the diffusion of singlet and triplet excitations relevant to TADF is definitively needed. This would require going beyond the widely used Förster model for weakly dipole-allowed CT singlets and including both exchange and superexchange interactions for triplets. ${ }^{99-101}$

- Highly emissive TADF emitters. Architectures sustaining multi-resonance effects ${ }^{28,102,103}$ have the potential to solve the conundrum of large singlet radiative decay rates despite small exchange energies. A proper description of the singlet and triplet excitations in these molecules calls for the inclusion of high-order electronic correlation effects, difficult to capture using conventional TD-DFT methods.

- Exciplexes. Despite considerable efforts to establish them as viable technology for OLED applications, exciplexes, namely emissive D-A intermolecular CT states, have been 
investigated in only very few computational studies. ${ }^{104,105}$ This is surely related to the weakness of intermolecular interactions in organics resulting in the large configurational space explored by the $\mathrm{D}$ and $\mathrm{A}$ molecules that can adopt multiple relative orientations. Besides sampling issues, another timely question relates to the quantum-mechanical effect driving RISC in exciplexes, with scenarios based on either spin-orbit or hyperfine field couplings proposed in the literature. ${ }^{104,106}$ Note that recent studies of crystalline multichromophoric materials, composed on a mixed stack of 1:1 D-A molecules, are particularly interesting in this context, too. ${ }^{107,108}$

As a last note, we would like to stress that, as highlighted in this perspective, modeling of TADF is a complex endeavor and we warn the community about the potential pitfalls of 'black box' calculations using standard approaches. While these might provide a useful first screening, we believe that the field of computational modeling has now reached a maturity level that allows for a truly firstprinciples description of TADF emitters and hope this perspective will guide modelers and experimentalists on finding their way to best practices in TADF.

\section{Biographical Sketches}

Yoann Olivier obtained a Ph.D. from the University of Mons in 2008. From 2009 to 2013, he held a postdoctoral fellowship from Belgian National Fund for Scientific Research (FNRS) and went on postdoctoral stays with Prof. Claudio Zannoni at the University of Bologna and Prof. Henning Sirringhaus at the University of Cambridge. He is a currently a research associate at the University of Mons His research interests deal with the understanding of electronic processes in organic conjugated and 2D materials, using a multiscale approach combining quantum-chemical methods, Monte Carlo approach and molecular dynamics simulations.

Juan-Carlos Sancho-Garcia obtained a Ph.D. in Quantum Chemistry in 2001 at the University of Alicante, followed by a postdoctoral stay (2002-2004) at the Laboratory for Chemistry of Novel Materials in Mons, Belgium. He returned to Alicante as a 'Ramon y Cajal' research fellow and received a permanent position in 2010 in the Dept. of Physical Chemistry. His work integrates the development of more accurate DFT methods with applications to the field of Organic Electronics to elucidate structure-property relationships." 
Luca Muccioli is assistant professor of Physical Chemistry at the University of Bologna (Italy). He earned a Ph.D. in Chemical Sciences from University of Bologna in 2003, under the supervision of Prof. Claudio Zannoni, with a thesis on atomistic computer simulations of liquid crystals.

From 2003 to 2014 he was postdoctoral researcher at the University of Bologna, and from 2014 to 2016 assistant professor at the Institut des Sciences Moléculaires of the University of Bordeaux (France). His research interests concern the application of computational chemistry techniques to the study of the physico-chemical properties of organic materials, with particular focus on the prediction of structural and electronic properties of liquid crystals and organic semiconductors through multiscale approaches

Gabriele D'Avino received his Ph.D. in Materials Science at the University of Parma in 2010. After a postdoctoral research position at the University of Bologna, and two Marie Curie fellowships at the University of Liege and at the University of Mons, he is currently CNRS associate researcher at the Institut Institut Néel, Grenoble. His research focuses on the multiscale modeling of organic functional materials, merging quantum and classical techniques.

David Beljonne got his PhD in Chemistry at the University of Mons-Hainaut in 1994. After postdoctoral stays at the Universities of Cambridge (with Prof. Friend) and Rochester (with Prof. Mukamel), he is now a Research Director of the Belgian National Science Foundation (FNRS) and Professor at the University of Mons. He is also a Visiting Principal Research Scientist at the Georgia Institute of Technology in Atlanta. His research activities deal with the modeling of semiconducting materials for energy applications.

\section{PULL QUOTES}

(i) Can we predict the nature of the excited states and tune the primary chemical structure of TADF emitters in order to maximize the efficiency of upconversion and light emission in TADF-based OLEDs?

(ii) How important is the simulation procedure in determining the final morphology, and in turn how does it influence the calculated electronic properties?

(iii) How can we design in silico TADF emitters that yield the right orientation in the solid-state matrix to maximize light outcoupling? 
(iv) How much torsional dynamics is affected by the host-guest interaction? How does it affect the efficiency of TADF?

(v) Polarization medium effects are stabilizing CT states with respect to LE states. Could we possibly target the right combination of host and guest in order to get negative $\Delta \mathrm{E}_{\mathrm{ST}}$ ?

(vi) Should we freeze-out the motion of triplet excitations in TADF materials in order to reduce annihilation processes?

\section{Acknowledgments}

The work in Mons was supported by the Belgian National Science Foundation, F.R.S.-FNRS. Computational resources have been provided by the Consortium des Équipements de Calcul Intensif (CÉCI), funded by F.R.S.-FNRS under Grant No. 2.5020.11 as well as the Tier-1 supercomputer of the Fédération Wallonie-Bruxelles, infrastructure funded by the Walloon Region under the grant agreement n1117545. The research in Bologna, Grenoble and Mons is also through the European Union's Horizon 2020 research and innovation program under Grant Agreement No. 646176 (EXTMOS project). GD would like to thank Prof. Xavier Blase for discussions. JC, LM and YO acknowledge discussion with Dr. Mónica Moral. DB and YO would like to thank Prof. Thuc Quyen Nguyen and Brett Yurash for the fruitful collaboration on the 2CzPN and 4CzIPN study. DB is a FNRS Research Director.

\section{References}

(1) Parker, C. A.; Hatchard, C. G. Triplet-Singlet Emission in Fluid Solutions. Phosphorescence of Eosin. Trans. Faraday Soc. 1961, 57, 1894-1904.

(2) Uoyama, H.; Goushi, K.; Shizu, K.; Nomura, H.; Adachi, C. Highly Efficient Organic LightEmitting Diodes from Delayed Fluorescence. Nature 2012, 492, 234-238.

(3) Etherington, M. K.; Gibson, J.; Higginbotham, H. F.; Penfold, T. J.; Monkman, A. P. Revealing the Spin-vibronic Coupling Mechanism of Thermally Activated Delayed Fluorescence. Nat. Commun. 2016, 7, 13680.

(4) Dias, F. B.; Santos, J.; Graves, D. R.; Data, P.; Nobuyasu, R. S.; Fox, M. A.; Batsanov, A. S.; Palmeira, T.; Berberan-Santos, M. N.; Bryce, M. R.; et al. The Role of Local Triplet Excited States and D-A Relative Orientation in Thermally Activated Delayed Fluorescence: Photophysics and Devices. Adv. Sci. 2016, 3, 1600080.

(5) Hosokai, T.; Matsuzaki, H.; Nakanotani, H.; Tokumaru, K.; Tsutsui, T.; Furube, A.; Nasu, K.; Nomura, H.; Yahiro, M.; Adachi, C. Evidence and Mechanism of Efficient Thermally Activated 
Delayed Fluorescence Promoted by Delocalized Excited States. Sci. Adv. 2017, 3, e1603282.

(6) Milián-Medina, B.; Gierschner, J. Computational Design of Low Singlet-triplet Gap All-Organic Molecules for OLED Application. Org. Electron. 2012, 13, 985-991.

(7) Olivier, Y.; Moral, M.; Muccioli, L.; Sancho-García, J.-C. Dynamic Nature of Excited States of Donor-Acceptor TADF Materials for OLEDs: How Theory Can Reveal Structure-Property Relationships. J. Mater. Chem. C 2017, 5, 5718-5729.

(8) Wong, M. Y.; Zysman-Colman, E. Purely Organic Thermally Activated Delayed Fluorescence Materials for Organic Light-Emitting Diodes. Adv. Mater. 2017, 29, 1605444.

(9) Schott, S.; McNellis, E. R.; Nielsen, C. B.; Chen, H. Y.; Watanabe, S.; Tanaka, H.; McCulloch, I.; Takimiya, K.; Sinova, J.; Sirringhaus, H. Tuning the Effective Spin-Orbit Coupling in Molecular Semiconductors. Nat. Commun. 2017, 8, 15200.

(10) Olivier, Y.; Yurash, B.; Muccioli, L.; D’Avino, G.; Mikhnenko, O.; Sancho-García, J. C.; Adachi, C.; Nguyen, T.-Q.; Beljonne, D. Nature of the Singlet and Triplet Excitations Mediating Thermally Activated Delayed Fluorescence. Phys. Rev. Mater. 2017, 1, 75602.

(11) Gómez-Bombarelli, R.; Aguilera-Iparraguirre, J.; Hirzel, T. D.; Duvenaud, D.; Maclaurin, D.; Blood-Forsythe, M. A.; Chae, H. S.; Einzinger, M.; Ha, D.-G.; Wu, T.; et al. Design of Efficient Molecular Organic Light-Emitting Diodes by a High-Throughput Virtual Screening and Experimental Approach. Nat. Mater. 2016, 15, 1120-1127.

Chen, X.-K.; Kim, D.; Brédas, J.-L. Thermally Activated Delayed Fluorescence (TADF) Path toward Efficient Electroluminescence in Purely Organic Materials: Molecular Level Insight. Acc. Chem. Res. 2018, 51, 2215-2224.

(13) Moral, M.; Muccioli, L.; Son, W.-J.; Olivier, Y.; Sancho-García, J. C. Theoretical Rationalization of the Singlet-Triplet Gap in OLEDs Materials: Impact of Charge-Transfer Character. J. Chem. Theory Comput. 2015, 11, 168-177.

(14) Sun, H.; Zhong, C.; Brédas, J. L. Reliable Prediction with Tuned Range-Separated Functionals of the Singlet-Triplet Gap in Organic Emitters for Thermally Activated Delayed Fluorescence. J. Chem. Theory Comput. 2015, 11, 3851-3858.

(15) Peng, Q.; Fan, D.; Duan, R.; Yi, Y.; Niu, Y.; Wang, D.; Shuai, Z. Theoretical Study of Conversion and Decay Processes of Excited Triplet and Singlet States in a Thermally Activated Delayed Fluorescence Molecule. J. Phys. Chem. C 2017, 121, 13448-13456.

(16) Schreiber, M.; Silva-Junior, M. R.; Sauer, S. P. A.; Thiel, W. Benchmarks for Electronically Excited States: CASPT2, CC2, CCSD, and CC3. J. Chem. Phys. 2008, 128, 134110.

(17) Silva-Junior, M. R.; Schreiber, M.; Sauer, S. P. A.; Thiel, W. Benchmarks for Electronically Excited States: Time-Dependent Density Functional Theory and Density Functional Theory Based Multireference Configuration Interaction. J. Chem. Phys. 2008, 129, 104103. 
(18) Jacquemin, D.; Wathelet, V.; Perpète, E. A.; Adamo, C. Extensive TD-DFT Benchmark: SingletExcited States of Organic Molecules. J. Chem. Theory Comput. 2009, 5, 2420-2435.

(19) Loos, P.-F.; Scemama, A.; Blondel, A.; Garniron, Y.; Caffarel, M.; Jacquemin, D. A Mountaineering Strategy to Excited States: Highly Accurate Reference Energies and Benchmarks. J. Chem. Theory Comput. 2018, 14, 4360-4379.

(20) Mewes, J.-M. Modeling TADF in Organic Emitters Requires a Careful Consideration of the Environment and Going beyond the Franck-Condon Approximation. Phys. Chem. Chem. Phys. 2018, 20, 12454-12469.

(21) Hellweg, A.; Grün, S. A.; Hättig, C. Benchmarking the Performance of Spin-Component Scaled CC2 in Ground and Electronically Excited States. Phys. Chem. Chem. Phys. 2008, 10, 41194127.

(22) Jacquemin, D.; Duchemin, I.; Blondel, A.; Blase, X. Benchmark of Bethe-Salpeter for Triplet Excited-States. J. Chem. Theory Comput. 2017, 13, 767-783.

(23) Noguchi, Y.; Sugino, O. High-Lying Triplet Excitons of Thermally Activated Delayed Fluorescence Molecules. J. Phys. Chem. C 2017, 121, 20687-20695.

(24) Rangel, T.; Hamed, S. M.; Bruneval, F.; Neaton, J. B. An Assessment of Low-Lying Excitation Energies and Triplet Instabilities of Organic Molecules with an Ab Initio Bethe-Salpeter Equation Approach and the Tamm-Dancoff Approximation. J. Chem. Phys. 2017, 146, 194108.

(25) Weigend, F.; Häser, M.; Patzelt, H.; Ahlrichs, R. RI-MP2: Optimized Auxiliary Basis Sets and Demonstration of Efficiency. Chem. Phys. Lett. 1998, 294, 143-152.

(26) Weigend, F. A Fully Direct RI-HF Algorithm: Implementation, Optimised Auxiliary Basis Sets, Demonstration of Accuracy and Efficiency. Phys. Chem. Chem. Phys. 2002, 4, 4285-4291.

(27) Werner, H.-J.; Manby, F. R.; Knowles, P. J. Fast Linear Scaling Second-Order Møller-Plesset Perturbation Theory (MP2) Using Local and Density Fitting Approximations. J. Chem. Phys. 2003, 118, 8149-8160.

(28) Hatakeyama, T.; Shiren, K.; Nakajima, K.; Nomura, S.; Nakatsuka, S.; Kinoshita, K.; Ni, J.; Ono, Y.; Ikuta, T. Ultrapure Blue Thermally Activated Delayed Fluorescence Molecules: Efficient HOMO-LUMO Separation by the Multiple Resonance Effect. Adv. Mater. 2016, 28, 2777-2781.

(29) Brückner, C.; Engels, B. Benchmarking Singlet and Triplet Excitation Energies of Molecular Semiconductors for Singlet Fission: Tuning the Amount of HF Exchange and Adjusting Local Correlation to Obtain Accurate Functionals for Singlet-triplet Gaps. Chem. Phys. 2017, 482, 319-338.

(30) Nakagawa, T.; Ku, S.-Y.; Wong, K.-T.; Adachi, C. Electroluminescence Based on Thermally Activated Delayed Fluorescence Generated by a Spirobifluorene Donor-acceptor Structure. Chem. Commun. 2012, 48, 9580-9582. 
(31) Cai, X.; Chen, D.; Gao, K.; Gan, L.; Yin, Q.; Qiao, Z.; Chen, Z.; Jiang, X.; Su, S.-J. "Trade-Off" Hidden in Condensed State Solvation: Multiradiative Channels Design for Highly Efficient Solution-Processed Purely Organic Electroluminescence at High Brightness. Adv. Funct. Mater. 2018, 28, 1704927.

(32) Huang, S.; Zhang, Q.; Shiota, Y.; Nakagawa, T.; Kuwabara, K.; Yoshizawa, K.; Adachi, C. Computational Prediction for Singlet- and Triplet-Transition Energies of Charge-Transfer Compounds. J. Chem. Theory Comput. 2013, 9, 3872-3877.

(33) Jacquemin, D.; Perpète, E. A.; Ciofini, I.; Adamo, C.; Valero, R.; Zhao, Y.; Truhlar, D. G. On the Performances of the M06 Family of Density Functionals for Electronic Excitation Energies. J. Chem. Theory Comput. 2010, 6, 2071-2085.

(34) Hirata, S.; Head-Gordon, M. Time-Dependent Density Functional Theory within the TammDancoff Approximation. Chem. Phys. Lett. 1999, 314, 291-299.

(35) Penfold, T. J. On Predicting the Excited-State Properties of Thermally Activated Delayed Fluorescence Emitters. J. Phys. Chem. C 2015, 119, 13535-13544.

(36) Leininger, T.; Stoll, H.; Werner, H.-J.; Savin, A. Combining Long-Range Configuration Interaction with Short-Range Density Functionals. Chem. Phys. Lett. 1997, 275, 151-160.

(37) likura, H.; Tsuneda, T.; Yanai, T.; Hirao, K. A Long-Range Correction Scheme for GeneralizedGradient-Approximation Exchange Functionals. J. Chem. Phys. 2001, 115, 3540-3544.

(38) Yanai, T.; Tew, D. P.; Handy, N. C. A New Hybrid Exchange-correlation Functional Using the Coulomb-Attenuating Method (CAM-B3LYP). Chem. Phys. Lett. 2004, 393, 51-57.

(39) Hait, D.; Zhu, T.; McMahon, D. P.; Van Voorhis, T. Prediction of Excited-State Energies and Singlet-Triplet Gaps of Charge-Transfer States Using a Restricted Open-Shell Kohn-Sham Approach. J. Chem. Theory Comput. 2016, 12, 3353-3359.

(40) Di, D.; Romanov, A. S.; Yang, L.; Richter, J. M.; Rivett, J. P. H.; Jones, S.; Thomas, T. H.; Abdi Jalebi, M.; Friend, R. H.; Linnolahti, M.; et al. High-Performance Light-Emitting Diodes Based on Carbene-Metal-Amides. Science 2017, 356, 159-163.

(41) Grimme, S.; Bannwarth, C. Ultra-Fast Computation of Electronic Spectra for Large Systems by Tight-Binding Based Simplified Tamm-Dancoff Approximation (sTDA-xTB). J. Chem. Phys. 2016, $145,054103$.

(42) Alipour, M.; Karimi, N. Dissecting the Accountability of Parameterized and Parameter-Free Single-Hybrid and Double-Hybrid Functionals for Photophysical Properties of TADF-Based OLEDs. J. Chem. Phys. 2017, 146, 234304.

(43) Lyskov, I.; Kleinschmidt, M.; Marian, C. M. Redesign of the DFT/MRCI Hamiltonian. J. Chem. Phys. 2016, 144, 034104.

(44) Föller, J.; Marian, C. M. Rotationally Assisted Spin-State Inversion in Carbene-Metal-Amides Is 
an Artifact. J. Phys. Chem. Lett. 2017, 8, 5643-5647.

(45) Taffet, E. J.; Olivier, Y.; Lam, F.; Beljonne, D.; Scholes, G. D. Carbene-Metal-Amide Bond Deformation, Rather Than Ligand Rotation, Drives Delayed Fluorescence. J. Phys. Chem. Lett.

2018, 9, 1620-1626.

(46) Maschietto, F.; Campetella, M.; Frisch, M. J.; Scalmani, G.; Adamo, C.; Ciofini, I. How Are the Charge Transfer Descriptors Affected by the Quality of the Underpinning Electronic Density? J. Comput. Chem. 2018, 39, 735-742.

(47) Peach, M. J. G.; Benfield, P.; Helgaker, T.; Tozer, D. J. Excitation Energies in Density Functional Theory: An Evaluation and a Diagnostic Test. J. Chem. Phys. 2008, 128, 044118.

(48) Peach, M. J. G.; Tozer, D. J. Illustration of a TDDFT Spatial Overlap Diagnostic by Basis Function Exponent Scaling. J. Mol. Struct. THEOCHEM 2009, 914, 110-114.

(49) Guido, C. A.; Cortona, P.; Mennucci, B.; Adamo, C. On the Metric of Charge Transfer Molecular Excitations: A Simple Chemical Descriptor. J. Chem. Theory Comput. 2013, 9, 3118-3126.

(50) Guido, C. A.; Cortona, P.; Adamo, C. Effective Electron Displacements: A Tool for TimeDependent Density Functional Theory Computational Spectroscopy. J. Chem. Phys. 2014, 140, 104101.

(51) Le Bahers, T.; Adamo, C.; Ciofini, I. A Qualitative Index of Spatial Extent in Charge-Transfer Excitations. J. Chem. Theory Comput. 2011, 7, 2498-2506.

(52) Etienne, T.; Assfeld, X.; Monari, A. Toward a Quantitative Assessment of Electronic Transitions' Charge-Transfer Character. J. Chem. Theory Comput. 2014, 10, 3896-3905.

(53) Campetella, M.; Maschietto, F.; Frisch, M. J.; Scalmani, G.; Ciofini, I.; Adamo, C. Charge Transfer Excitations in TDDFT: A Ghost-Hunter Index. J. Comput. Chem. 2017, 38, 2151-2156.

(54) Savarese, M.; Guido, C. A.; Brémond, E.; Ciofini, I.; Adamo, C. Metrics for Molecular Electronic Excitations: A Comparison between Orbital- and Density-Based Descriptors. J. Phys. Chem. A 2017, 121, 7543-7549.

(55) Etienne, T.; Assfeld, X.; Monari, A. New Insight into the Topology of Excited States through Detachment/Attachment Density Matrices-Based Centroids of Charge. J. Chem. Theory Comput. 2014, 10, 3906-3914.

(56) Samanta, P. K.; Kim, D.; Coropceanu, V.; Brédas, J. L. Up-Conversion Intersystem Crossing Rates in Organic Emitters for Thermally Activated Delayed Fluorescence: Impact of the Nature of Singlet vs Triplet Excited States. J. Am. Chem. Soc. 2017, 139, 4042-4051.

(57) Köhler, A.; Beljonne, D. The Singlet-Triplet Exchange Energy in Conjugated Polymers. Adv. Funct. Mater. 2004, 14, 11-18.

(58) Richert, S.; Tait, C. E.; Timmel, C. R. Delocalisation of Photoexcited Triplet States Probed by Transient EPR and Hyperfine Spectroscopy. J. Magn. Reson. 2017, 280, 103-116. 
(59) Barone, V. Structure, Magnetic Properties and Reactivities of Open-Shell Species From Density Functional and Self-Consistent Hybrid Methods. In Recent Advances in Computational Chemistry; 1995; pp. 287-334.

(60) Evans, E. W.; Olivier, Y.; Puttisong, Y.; Myers, W. K.; Hele, T. J. H.; Menke, S. M.; Thomas, T. H.; Credgington, D.; Beljonne, D.; Friend, R. H.; et al. Vibrationally Assisted Intersystem Crossing in Benchmark Thermally Activated Delayed Fluorescence Molecules. J. Phys. Chem. Lett. 2018, 9, 4053-4058.

(61) Chen, X.-K.; Tsuchiya, Y.; Ishikawa, Y.; Zhong, C.; Adachi, C.; Brédas, J.-L. A New Design Strategy for Efficient Thermally Activated Delayed Fluorescence Organic Emitters: From Twisted to Planar Structures. Adv. Mater. 2017, 29, 1702767.

(62) Walker, T. E. H.; Richards, W. G. Molecular Spin-Orbit Coupling Constants. The Role of Core Polarization. J. Chem. Phys. 1970, 52, 1311-1314.

(63) Alberto, M. E.; De Simone, B. C.; Mazzone, G.; Quartarolo, A. D.; Russo, N. Theoretical Determination of Electronic Spectra and Intersystem Spin-Orbit Coupling: The Case of Isoindole-BODIPY Dyes. J. Chem. Theory Comput. 2014, 10, 4006-4013.

(64) Lower, S. K.; El-Sayed, M. A. The Triplet State and Molecular Electronic Processes in Organic Molecules. Chem. Rev. 1966, 66, 199-241.

(65) M. Klessinger, J. M. Excited States and Photochemistry of Organic Molecules; VCH Weinheim, 1995.

(66) Gibson, J.; Penfold, T. J. Nonadiabatic Coupling Reduces the Activation Energy in Thermally Activated Delayed Fluorescence. Phys. Chem. Chem. Phys. 2017, 19, 8428-8434.

(67) Fan, D.; Yi, Y.; Li, Z.; Liu, W.; Peng, Q.; Shuai, Z. Solvent Effects on the Optical Spectra and Excited-State Decay of Triphenylamine-Thiadiazole with Hybridized Local Excitation and Intramolecular Charge Transfer. J. Phys. Chem. A 2015, 119, 5233-5240.

(68) Levine, B. G.; Martínez, T. J. Isomerization Through Conical Intersections. Annu. Rev. Phys. Chem. 2007, 58, 613-634.

(69) Blancafort, L. Photochemistry and Photophysics at Extended Seams of Conical Intersection. ChemPhysChem 2014, 15, 3166-3181.

(70) Murawski, C.; Leo, K.; Gather, M. C. Efficiency Roll-Off in Organic Light-Emitting Diodes. Adv. Mater. 2013, 25, 6801-6827.

(71) Smith, M. B.; Michl, J. Singlet Fission. Chem. Rev. 2010, 110, 6891-6936.

(72) Coehoorn, R.; van Eersel, H.; Bobbert, P.; Janssen, R. Kinetic Monte Carlo Study of the Sensitivity of OLED Efficiency and Lifetime to Materials Parameters. Adv. Funct. Mater. 2015, 25, 2024-2037.

(73) Muccioli, L.; D’Avino, G.; Berardi, R.; Orlandi, S.; Pizzirusso, A.; Ricci, M.; Roscioni, O. M.; 
Zannoni, C. Supramolecular Organization of Functional Organic Materials in the Bulk and at Organic/Organic Interfaces: A Modeling and Computer Simulation Approach. Top. Curr. Chem. 2013, 352, 39-101.

(74) Groves, C. Simulating Charge Transport in Organic Semiconductors and Devices: A Review. Rep. Prog. Phys. 2017, 80, 026502.

(75) Northey, T.; Stacey, J.; Penfold, T. J. The Role of Solid State Solvation on the Charge Transfer State of a Thermally Activated Delayed Fluorescence Emitter. J. Mater. Chem. C 2017, 5, 11001-11009.

(76) Muccioli, L.; D’Avino, G.; Zannoni, C. Simulation of Vapor-Phase Deposition and Growth of a Pentacene Thin Film on C60 (001). Adv. Mater. 2011, 23, 4532-4536.

(77) D’Avino, G.; Muccioli, L.; Zannoni, C. From Chiral Islands to Smectic Layers: A Computational Journey Across Sexithiophene Morphologies on C 60. Adv. Funct. Mater. 2015, 25, 1985-1995.

(78) Dalal, S. S.; Walters, D. M.; Lyubimov, I.; de Pablo, J. J.; Ediger, M. D. Tunable Molecular Orientation and Elevated Thermal Stability of Vapor-Deposited Organic Semiconductors. Proc. Natl. Acad. Sci. USA 2015, 112, 4227-4232.

(79) Friederich, P.; Coehoorn, R.; Wenzel, W. Molecular Origin of the Anisotropic Dye Orientation in Emissive Layers of Organic Light Emitting Diodes. Chem. Mater. 2017, 29, 9528-9535.

(80) Tonnelé, C.; Stroet, M.; Caron, B.; Clulow, A. J.; Nagiri, R. C. R.; Malde, A. K.; Burn, P. L.; Gentle, I. R.; Mark, A. E.; Powell, B. J. Elucidating the Spatial Arrangement of Emitter Molecules in Organic Light-Emitting Diode Films. Angew. Chemie Int. Ed. 2017, 56, 8402-8406.

(81) Youn, Y.; Yoo, D.; Song, H.; Kang, Y.; Kim, K. Y.; Jeon, S. H.; Cho, Y.; Chae, K.; Han, S. All-Atom Simulation of Molecular Orientation in Vapor-Deposited Organic Light-Emitting Diodes. J. Mater. Chem. C 2018, 6, 1015-1022.

(82) Shibata, M.; Sakai, Y.; Yokoyama, D. Advantages and Disadvantages of Vacuum-Deposited and Spin-Coated Amorphous Organic Semiconductor Films for Organic Light-Emitting Diodes. J. Mater. Chem. C 2015, 3, 11178-11191.

(83) Ediger, M. D. Perspective: Highly Stable Vapor-Deposited Glasses. J. Chem. Phys. 2017, 147, 210901.

(84) Komino, T.; Tanaka, H.; Adachi, C. Selectively Controlled Orientational Order in Linear-Shaped Thermally Activated Delayed Fluorescent Dopants. Chem. Mater. 2014, 26, 3665-3671.

(85) Hasegawa, Y.; Yamada, Y.; Sasaki, M.; Hosokai, T.; Nakanotani, H.; Adachi, C. Well-Ordered 4CzIPN ((4s,6s)-2,4,5,6-Tetra(9-H-Carbazol-9-YI)isophthalonitrile) Layers: Molecular Orientation, Electronic Structure, and Angular Distribution of Photoluminescence. J. Phys. Chem. Lett. 2018, 9, 863-867.

(86) Schmidt, T. D.; Lampe, T.; Sylvinson M. R., D.; Djurovich, P. I.; Thompson, M. E.; Brütting, W. 
Emitter Orientation as a Key Parameter in Organic Light-Emitting Diodes. Phys. Rev. Appl. 2017, 8, 037001.

(87) Mayr, C.; Lee, S. Y.; Schmidt, T. D.; Yasuda, T.; Adachi, C.; Brütting, W. Efficiency Enhancement of Organic Light-Emitting Diodes Incorporating a Highly Oriented Thermally Activated Delayed Fluorescence Emitter. Adv. Funct. Mater. 2014, 24, 5232-5239.

(88) Lyubimov, I.; Antony, L.; Walters, D. M.; Rodney, D.; Ediger, M. D.; de Pablo, J. J. Orientational Anisotropy in Simulated Vapor-Deposited Molecular Glasses. J. Chem. Phys. 2015, 143, 094502.

(89) Alessandri, R.; Uusitalo, J. J.; de Vries, A. H.; Havenith, R. W. A.; Marrink, S. J. Bulk Heterojunction Morphologies with Atomistic Resolution from Coarse-Grain Solvent Evaporation Simulations. J. Am. Chem. Soc. 2017, 139, 3697-3705.

(90) Moral, M.; Son, W.-J.; Sancho-García, J. C.; Olivier, Y.; Muccioli, L. Cost-Effective Force Field Tailored for Solid-Phase Simulations of OLED Materials. J. Chem. Theory Comput. 2015, 11, 3383-3392.

(91) Andreussi, O.; Prandi, I. G.; Campetella, M.; Prampolini, G.; Mennucci, B. Classical Force Fields Tailored for QM Applications: Is It Really a Feasible Strategy? J. Chem. Theory Comput. 2017, $13,4636-4648$.

(92) Chen, X. K.; Zhang, S. F.; Fan, J. X.; Ren, A. M. Nature of Highly Efficient Thermally Activated Delayed Fluorescence in Organic Light-Emitting Diode Emitters: Nonadiabatic Effect between Excited States. J. Phys. Chem. C 2015, 119, 9728-9733.

(93) Wang, L.; Prezhdo, O. V.; Beljonne, D. Mixed Quantum-Classical Dynamics for Charge Transport in Organics. Phys. Chem. Chem. Phys. 2015, 17, 12395-12406.

(94) Meyer, H.-D.; Manthe, U.; Cederbaum, L. S. The Multi-Configurational Time-Dependent Hartree Approach. Chem. Phys. Lett. 1990, 165, 73-78.

(95) Coropceanu, V.; Sánchez-Carrera, R. S.; Paramonov, P.; Day, G. M.; Brédas, J.-L. Interaction of Charge Carriers with Lattice Vibrations in Organic Molecular Semiconductors: Naphthalene as a Case Study. J. Phys. Chem. C 2009, 113, 4679-4686.

(96) Marian, C. M. Mechanism of the Triplet-to-Singlet Upconversion in the Assistant Dopant ACRXTN. J. Phys. Chem. C 2016, 120, 3715-3721.

(97) Sun, H.; Hu, Z.; Zhong, C.; Chen, X.; Sun, Z.; Brédas, J.-L. Impact of Dielectric Constant on the Singlet-Triplet Gap in Thermally Activated Delayed Fluorescence Materials. J. Phys. Chem. Lett. 2017, 8, 2393-2398.

(98) Nakanotani, H.; Higuchi, T.; Furukawa, T.; Masui, K.; Morimoto, K.; Numata, M.; Tanaka, H.; Sagara, Y.; Yasuda, T.; Adachi, C. High-Efficiency Organic Light-Emitting Diodes with Fluorescent Emitters. Nat. Commun. 2014, 5, 4016. 
(99) Dexter, D. L. A Theory of Sensitized Luminescence in Solids. J. Chem. Phys. 1953, 21, 836-850.

(100) McConnell, H. M. Intramolecular Charge Transfer in Aromatic Free Radicals. J. Chem. Phys. 1961, 35, 508-515.

(101) Beljonne, D.; Curutchet, C.; Scholes, G. D.; Silbey, R. J. Beyond Förster Resonance Energy Transfer in Biological and Nanoscale Systems. J. Phys. Chem. B 2009, 113, 6583-6599.

(102) Nakatsuka, S.; Gotoh, H.; Kinoshita, K.; Yasuda, N.; Hatakeyama, T. Divergent Synthesis of Heteroatom-Centered 4,8,12-Triazatriangulenes. Angew. Chemie Int. Ed. 2017, 56, 50875090.

(103) Matsui, K.; Oda, S.; Yoshiura, K.; Nakajima, K.; Yasuda, N.; Hatakeyama, T. One-Shot Multiple Borylation toward BN-Doped Nanographenes. J. Am. Chem. Soc. 2018, 140, 1195-1198.

(104) Hontz, E.; Chang, W.; Congreve, D. N.; Bulović, V.; Baldo, M. A.; Van Voorhis, T. The Role of Electron-Hole Separation in Thermally Activated Delayed Fluorescence in Donor-Acceptor Blends. J. Phys. Chem. C 2015, 119, 25591-25597.

(105) Huang, Y.; Westenhoff, S.; Avilov, I.; Sreearunothai, P.; Hodgkiss, J. M.; Deleener, C.; Friend, R. H.; Beljonne, D. Electronic Structures of Interfacial States Formed at Polymeric Semiconductor Heterojunctions. Nat. Mater. 2008, 7, 483-489.

(106) Wang, Y.; Sahin-Tiras, K.; Harmon, N. J.; Wohlgenannt, M.; Flatté, M. E. Immense Magnetic Response of Exciplex Light Emission due to Correlated Spin-Charge Dynamics. Phys. Rev. $X$ 2016, 6, 11011.

(107) Wykes, M.; Park, S. K.; Bhattacharyya, S.; Varghese, S.; Kwon, J. E.; Whang, D. R.; Cho, I.; Wannemacher, R.; Lüer, L.; Park, S. Y.; et al. Excited State Features and Dynamics in a Distyrylbenzene-Based Mixed Stack Donor-Acceptor Cocrystal with Luminescent Charge Transfer Characteristics. J. Phys. Chem. Lett. 2015, 6, 3682-3687.

(108) Wykes, M.; Parambil, R.; Beljonne, D.; Gierschner, J. Vibronic Coupling in Molecular Crystals: A Franck-Condon Herzberg-Teller Model of H-Aggregate Fluorescence Based on Quantum Chemical Cluster Calculations. J. Chem. Phys. 2015, 143, 114116. 
1

2

3

4

5

6

7

8

9

\section{ACRONYMS}

OLED:

Organic Light Emitting Diode

TADF: $\quad$ Thermally Activated Delayed Fluorescence

IQE: Internal Quantum Efficiency

$\mathrm{T}_{\mathrm{n}}: \quad \mathrm{n}$-th triplet excited state

$\mathrm{S}_{\mathrm{n}}: \quad \mathrm{n}$-th singlet excited state

(R)ISC: $\quad$ (Reverse) InterSystem Crossing

D: $\quad$ Electron donating moiety/unit

A: $\quad$ Electron accepting moiety/unit

CT: $\quad$ Charge Transfer

LE: $\quad$ Local Excitation

DFT: Density Functional Theory

TD-DFT: $\quad$ Time-Dependent Density Functional Theory

PCM: $\quad$ polarizable continuum models^

$\Delta E_{S T}^{V}: \quad$ vertical exchange gap/vertical singlet-triplet energy gap

$\Omega\left(\mathrm{S}_{1}\right): \quad$ Lowest singlet excited state energy

$\Omega\left(T_{1}\right): \quad$ Lowest triplet excited state energy

CCSDT: $\quad$ Coupled-Cluster with Single, Double, and (iterative) Triple substitutions

CC3: $\quad$ Third-Order Approximate Coupled-Cluster

EOM-CCSD: Equation-Of-Motion Coupled-Cluster with Single and Double substitutions

$\mathrm{FCl} \quad \quad \quad \quad \quad$ Full Configuration Interactions

CC2: $\quad$ Second-Order Approximate Coupled-Cluster

ADC(2): $\quad$ Algebraic Diagrammatic Construction at second-order

$A D C(3): \quad \quad \quad \quad$ Algebraic Diagrammatic Construction at third-order

ADC(2)-x: $\quad$ eXtended Algebraic Diagrammatic Construction at second-order

RAS: $\quad$ Restricted Active Space

CASSCF: $\quad$ Complete Active Space Self-Consistent-Field

CASPT2: Complete Active Space Perturbation Theory at second-order

GW+BSE: Green's functions with Bethe-Salpeter Equation

B3LYP: $\quad$ Becke three-parameter hybrid Lee-Yang-Parr exchange-correlation functional

6-31G*: $\quad$ Pople's double- $\xi$ basis set with polarization functions on 2 nd row atoms

M06-2X: $\quad$ Minnesota exchange-correlation functional in its 2006 version

TDA-DFT: Time-Dependent Density Functional Theory in the Tamm-Dancoff approximation

CIS: $\quad$ Configuration Interaction with Single substitutions 
TD-HF: $\quad$ Time-Dependent Hartree-Fock

PBE0: $\quad$ Perdew-Burke-Ernzerhof one-parameter hybrid exchange-correlation functional

D3(BJ): Dispersion correction (third-generation) with Becke-Johnson attenuation function

def2-TZVP: Alhrichs' triple- $\xi$ valence polarization basis set extended with diffuse function

CAM-B3LYP: Coulomb-attenuating method B3LYP exchange-correlation functional

$\omega B$ 97X: $\quad \omega$-dependent range-separated Becke'97 exchange-correlation functional

HOMO: Highest-Occupied Molecular Orbital

LUMO: $\quad$ Lowest-Unoccupied Molecular Orbital

UKS: $\quad$ Unrestricted Kohn-Sham

ROKS: $\quad$ Restricted Open-Shell Kohn-Sham

STDA-DFT: $\quad$ Simplified Tamm-Dancoff Time-Dependent Density Functional Theory

STDA-xTB: $\quad$ Simplified Tamm-Dancoff Extended Tight-Binding Hamiltonian

MP2: $\quad$ Second order Møller-Plesset perturbation method

MRCI: Multi-Reference Configuration Interaction

KS: $\quad$ Kohn-Sham orbitals

NTO: $\quad$ Natural Transition Orbitals

$\Delta \mathrm{E}_{\mathrm{ST}}: \quad$ Singlet-triplet energy gap

ZFS: $\quad$ Zero-Field Splitting

ESR: $\quad$ Electron Spin Resonance

2CzPN: 4,5-di(9h-carbazol-9-yl)phthalonitrile

4CzIPN: 1,2,3,5-tetrakis(carbazol-9-yl)-4,6-dicyanobenzene

(R)IC: $\quad$ (Reverse) Internal Conversion

MD: $\quad$ Molecular Dynamics

MC: $\quad$ Monte Carlo

QM: Quantum mechanical

$\mathrm{S}_{0}$ : $\quad$ Ground state

${ }^{3} \mathrm{CT}$ : Triplet charge transfer state

${ }^{3} \mathrm{LE}: \quad$ Triplet local excitation

$\varepsilon: \quad$ Dielectric constant

PXZ-OXD : Phenoxazine- 2,5-diphenyl-1,3,4-oxadiazole

TXO-TPA: 2- [4- (diphenylamino) phenyl] - 10, 10- dioxide-9H - thioxanthen-9- one 
TXO-PhCz: 2- (9- phenyl- 9H-carbazol-3-yl)-10,10- dioxide-9H-thioxanthen-9- one 